\title{
偶氮二甲酸酯在有机合成中的研究进展
}

\author{
许孝良* 程国华李小年* \\ (浙江工业大学化学工程与材料学院 杭州 310032)
}

\begin{abstract}
摘要 偶氮二甲酸酯由于具有独特的电子和结构特性, 作为一种多功能化的试剂在有机合成中得到了广泛的应用. 特 别是在 Mitsunobu 反应、羰基化合物的氨化、不饱烃的氨化及杂环化合物的合成中有着非常重要的作用，此外偶氮二 甲酸酯还可以作为氧化剂, 用于醇、胺的脱氢氧化反应. 回顾了其在有机合成中原有的应用基础之上, 对其近年来在合 成化学中的新发展进行了综述, 并对其发展加以展望.
\end{abstract}

关键词 偶氮二甲酸酯; 有机合成; 碳氮键形成

\section{Application of Azodicarboxylates in Organic Synthesis}

\author{
$\mathrm{Xu}$, Xiaoliang* Cheng, Guohua Li, Xiaonian* \\ (College of Chemical Engineering and Material Science, Zhejiang University of Technology, Hangzhou 310032)
}

\begin{abstract}
Azodicarboxylates, as versatile reagents, have been widely used in organic synthesis due to their unique electronic and structural properties. They have played important roles in organic synthesis such as Mitsunobu reaction, amination of carbonyl compounds and unsaturated hydrocarbons, synthesis of heterocyclic compounds. Besides these, azodicarboxylates can be used as oxidants for the dehydrogenation of alcohols and amines. Based on their previous applications, new progress of azodicarboxylates in synthetic chemistry in recent years is summarized and an outlook on their development is also given.
\end{abstract}

Keywords azodicarboxylates; organic synthesis; $\mathrm{C}-\mathrm{N}$ bond formation

偶氮二甲酸酯是一类同时带有偶氮和羧基官能团 的化合物, 比较常见的有偶氮二甲酸二甲酯(DMAD)、 偶氮二甲酸二乙酯(DEAD)、偶氮二甲酸二异丙酯 (DIAD)、偶氮二甲酸二叔丁酯(DTBAD)、偶氮二甲酸二 芐酯(DBAD)、偶氮二甲酸二(三氯乙基)酯(BTCEAD) (Scheme 1).

偶氮二甲酸二烷基酯又被称为 Mitsunobu 试剂, 是 Mitsunobu 反应(Eq. 1)中重要的试剂. Mitsunobu 反应由 Mitsunobu $^{[1]}$ 在 20 世纪 60,70 年代发现. 在 DEAD 与 $\mathrm{Ph}_{3} \mathrm{P}(3)$ 存在下伯醇或仲醇与亲核试剂发生取代反应, 最后会得到构型翻转的产物, 因此在有机合成及药物合 成中有非常重要的用途. 目前已有多篇关于 Mitsunobu 反应的评论与综述 ${ }^{[2]}$, 在此不再赘述. 由于偶氮二甲酸 酯的偶氮双键的缺电性, 常作为原料参与反应, 在形成 碳氮键的反应中是非常好的氮源; 除此之外还可参与自 由基反应、成环反应等.

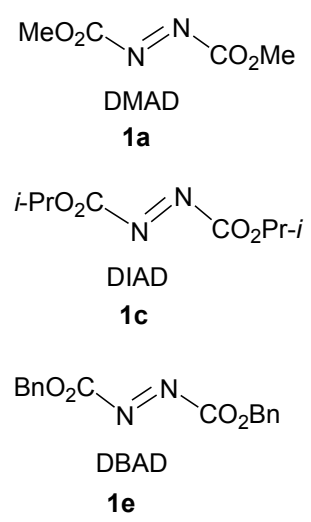

\section{Scheme 1}

\section{1 氨化反应}

\section{1 醛、酮的氨化}

在有机化学及生物化学中碳氮键在各类活性分子 中都广泛存在，碳氮键的合成也一直是有机化学家热衷

\footnotetext{
*E-mail: xuxiaoliang@zjut.edu.cn; xnli@zjut.edu.cn

Received October 31, 2011; revised December 16, 2011; published online January 6, 2012.

Project supported by the National Natural Science Foundation of China (No. 20872131).

国家自然科学基金(No. 20872131)资助项目.
} 


$$
\begin{aligned}
& \mathrm{R}^{1}{\stackrel{\mathrm{R}}{\mathrm{R}^{2}}}_{1}+\mathrm{H}-\mathrm{Nu}+1 \mathrm{~b}+\mathrm{PPh}_{3} \stackrel{\text { solvent }}{\longrightarrow} \\
& 1^{0} \text { or } 2^{0} \text { alcohol }
\end{aligned}
$$

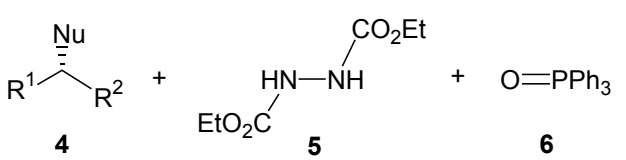

的课题, 羰基化合物的氨化反应是合成碳氮键的有效合 成路线, 通过此方法可以合成 $\alpha$-氨基醛、 $\alpha$-氨基醇、 $\alpha$ 氨基酸等多种生物活性分子。使用偶氮二甲酸酯作为氮 源进行氨化反应, 可以追溯到 1954 年 Huisgen 等 ${ }^{[3 a]}$ 使用 环己酮与 DEAD 在 $\mathrm{H}_{2} \mathrm{SO}_{4}$ 或 KOAc 的催化下的偶联反 应. 在二异丙基氨基锂等化合物的存在下将羰基化合物 烯醇化后，与偶氮二甲酸酯反应得 $\alpha$-氨基酸和 $\alpha$-肼基 酸, 也有多位化学家予以报道过 ${ }^{[3 b \sim 3 d]} .1997$ 年 Evans 及 其合作 者 ${ }^{[3 \mathrm{c}, 3 \mathrm{e}]}$ 还报道了醇钠、醇锞也同样可以高效催 化该反应, 并发现 $N$-酰基噁唑烷酩氨化的产物 $\mathbf{8 b}$ 也可 用作催化剂实现 $N$-酰基噁唑烷酮的氨化(Eq. 2).

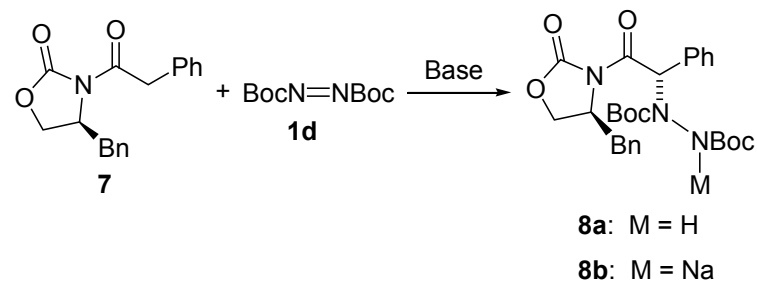

根据这一现象 Evans 等使用手性二胺与金属镁衍生 出双磺酰胺镁络合物 10 (Eq. 3).
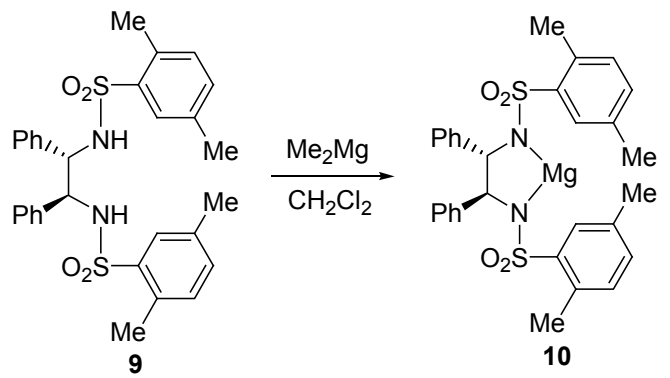

络合物 10 也可催化 Eq. 2 反应产率有 $90 \%$ 以上, ee 值高达 99\%. Evans 及其合作者还曾报道了在手性金属 催化剂 11(双噁唑啉铜, 见酮酯的氨化)存在下催化已烯 醇化的芳酮与非对称的偶氮二甲酸酯 13 的氨化反应 (Eq. 4). 该催化剂不仅对芳基直链酮有高的催化剂活性, 同时对环酮、缩醛、酰基吡咯同样有效 ${ }^{[3 f]}$.

2000 年 Yamashita 及其合作者 ${ }^{[3 \mathrm{~g}]}$ 用银盐作催化剂作 了类似的研究, $\mathrm{AgOTf}$ 和 $\mathrm{AgClO}_{4}$-BINAP 都适合烯醇醚 与偶氮二甲酸酯的氨化反应.
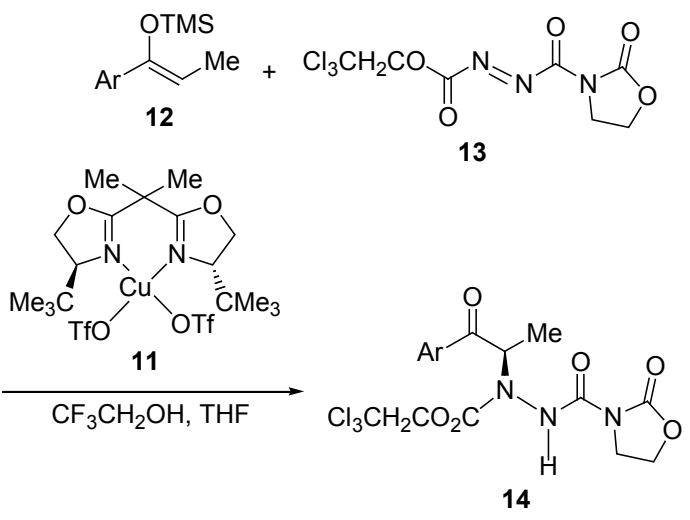

与上述非直接氨化反应相比不同, 2002 年 $\mathrm{List}^{[4 \mathrm{a}]}$ 和 Jørgensen 课题组 ${ }^{[4 b]}$ 分别独立地报道了使用脯氨酸 15 作 催化剂实现醛的直接不对称氨化反应(Eq. 5), 这提供了 另一条简易的合成含 $\alpha$-氨基醛、 $\alpha$-氨基醇、 $\alpha$-氨基酸光 学活性分子的路径. 由于不用将羰基化合物事先烯醇 化，因此更符合原子经济性原则.

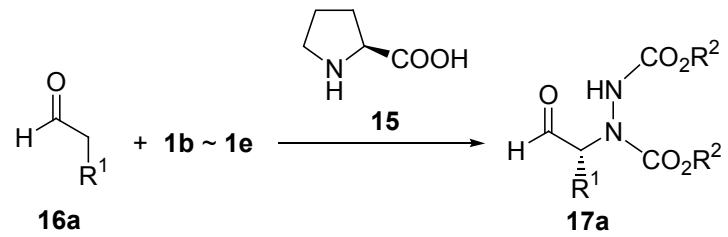

不久 Jørgensen 课题组 ${ }^{[4 \mathrm{c}]}$ 就将此方法拓展到脂肪族 直链酩和环己酮的不对称氨化作用(Eq. 6).

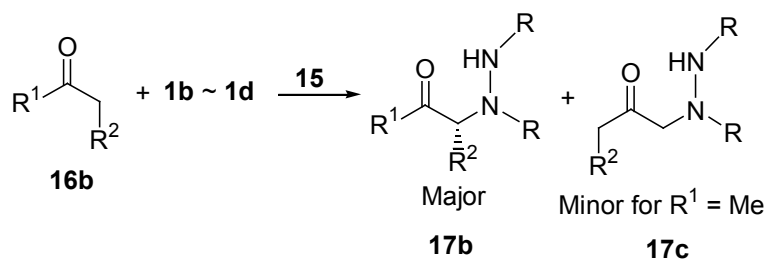

在脂肪酮的氨化中，若酮是不对称酮，氨化选择性 地发生在含取代基较多的 $\alpha$-碳上. 芳基酮的氨化反应相 对较少, 考虑到空间位阻的影响, Chen 及其合作者 ${ }^{[4 \mathrm{~d}]}$ 认 为伯胺相对于仲胺更适合于芳酮的对映选择性氨化反 应，他们研究了金鸡纳碱衍生的衍生物 18 作催化剂对 芳基酮的不对称催化氨化反应(Eq. 7), 反应对多种底物 都能适用，对映选择性可达 $98 \%$, 但反应所用的催化剂 用量相对较大 $(20 \mathrm{~mol} \%)$.

尽管 Jørgensen 和 Chen 等对酩的氨化作了报道, 但 除环己酮外对其它环状脂肪酤的直接氨化却鲜有报道. 2008 年 Hayashi 等 ${ }^{[4 \mathrm{e}]}$ 报道了使用硅氧脯氨酸 19 作催化 剂成功地实现环状脂肪酮与 DBAD 的氨化反应(Eq. 8), 而母体脯氨酸对此反应的催化效果则不理想，使得羰基 化合物的 $\alpha$-氨化反应的应用范围得到拓展. 


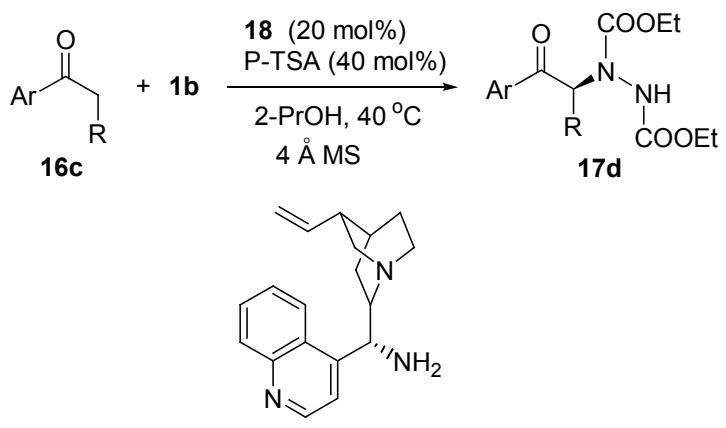

18

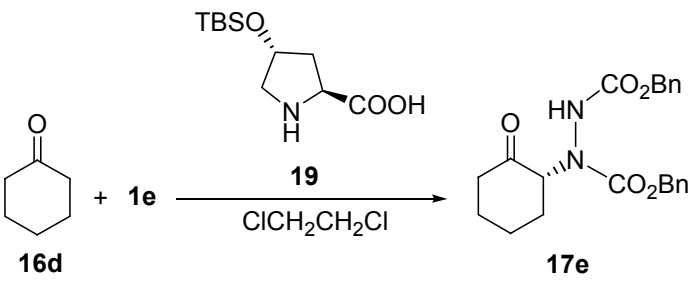

(8)

在 Jørgensen 和 List 的研究之后, Brase 等 ${ }^{[4 \sim 4 \mathrm{~g}]}$ 报道 了用脯氨酸催化 $\alpha, \alpha$-双取代外消旋醛的氨化反应(Eq. 9).<smiles>[R]C([Y16])C([R])C=O</smiles>

将反应体系置于微波条件下反应也能顺利发生，且 产率更高, $e e$ 值也明显提高 ${ }^{[4 h]}$, 同时利用 $\alpha, \alpha$-双取代醛 的氨化可以合成気代和氟代的 $\alpha, \alpha$-双取代酸衍生物 ${ }^{[4 \mathrm{i}]}$.

2003 年 Barbas 等 ${ }^{[4 j]}$ 报道了脯氨酸催化的醛、酮、 偶氮二甲酸酯的三组份一锅反应(Eq. 10), 这是一个典 型的醛酩作供体的组装反应(assembly reaction), 该方法 可合成 $\beta$-氨基醇.

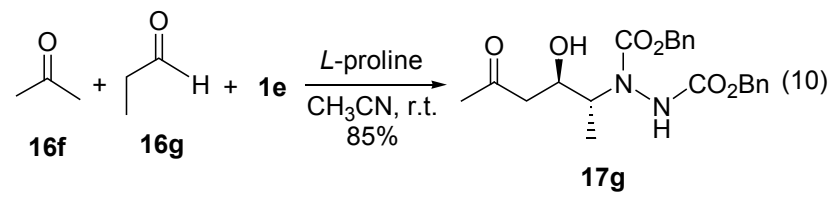

不久 Barbas 等 ${ }^{[4 k]}$ 成功将醛的氨化应用于细胞粘合 抑制剂 BRIT-337 (20)的全合成当中(Scheme 2).

AIDA (22), APICA (23)和多种神经系统疾病有关, 并有可能成为治疗此类疾病的药物. Barbas 等 ${ }^{[41]}$ 利用醛 的氨化合成 $\alpha$-肼基醛, 再将产物氧化和 $\mathrm{N}-\mathrm{N}$ 键解离成 功制得了 AIDA 和 APICA (Scheme 3).

使用脯氨酸作催化剂虽是非常好的反应, 但由于脯 氨酸的溶解性较差, 在反应体系中催化剂的量往往较大 $(10 \sim 20 \mathrm{~mol} \%)$, 因此转换率相对较低. Toma 等 ${ }^{[4 \mathrm{~m}]}$ 发现 离子液体 $\left([\mathrm{bmim}] \mathrm{BF}_{4}\right)$ 是非常好的溶剂, 可用于脯氨酸 催化的醛的氨化反应, 脯氨酸的用量可降低到 $5 \mathrm{~mol} \%$.

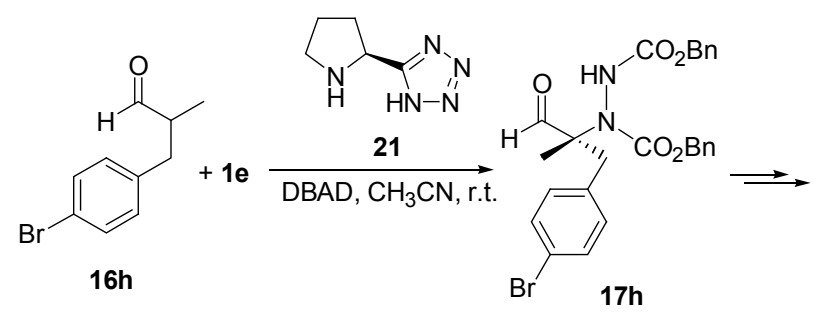<smiles>CN1C(=O)N(c2cc(Cl)cc(Cl)c2)C(=O)[C@]1(C)Cc1ccc(Br)cc1</smiles>

Scheme 2

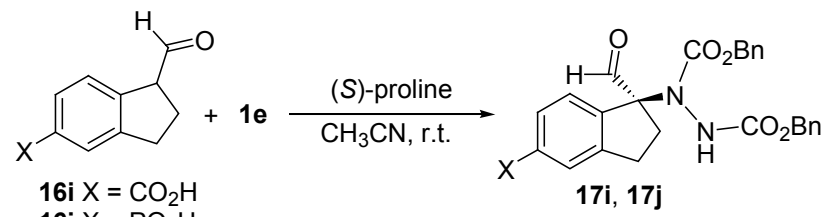

16j $\mathrm{X}=\mathrm{PO}_{3} \mathrm{H}_{2}$

$$
\text { (1) Oxidation } \mathrm{N-N \text {cleavage }}
$$

Scheme 3

由于醛酮的氨化反应在有机合成是非常重要的反 应，对催化剂的选择也吸引了诸多化学家的兴趣. 脯氨 酸衍生物及同系物如 $N$-芳磺酰基-2-氨基甲基吡咯烷 ${ }^{[5 a}$, $\alpha, \alpha$-二芳基甲硅烷基吡咯烷衍生物 ${ }^{[5 b]}$, 脯氨酸咪唑盐 ${ }^{[5 c]}$,

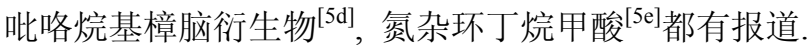

Toma 和 Wills 等报道了噻唑甲酸 ${ }^{[4 \mathrm{~m}]}$ 、缩醛胺 ${ }^{[4 \mathrm{~m}, 5 \mathrm{f}]}$ 均可用于醛与偶氮二甲酸酯的氨化反应.

双官能团催化剂可同时活化反应中的两个分子在 不对称催化中有广泛应用, Wang 和 $\mathrm{Xu}$ 课题组 ${ }^{[5 \mathrm{~g} \sim 5 \mathrm{~h}]}$ 首次 将仲胺硫嫝成功用于支链醛与偶氮二甲酸酯的氨化反 应，其中使用催化剂 24 可使产物的产率和对映选择性 达到 $97 \%$ 和 $99 \%$ (Eq. 11).

最近 Wang 和 $\mathrm{Xu}^{[5 i]}$ 以及 $\mathrm{Lu}$ 等 ${ }^{[5 \mathrm{j}]}$ 分别报道了使用手 性伯氨基酸以及离子对催化剂直接氨化支链醛.

Hamada 等 $^{[5 \mathrm{k}]}$ 报道了 $\beta$-氨基醛氨化制得 2,3 -二氨基 丁酸衍生物 $\mathbf{1 7 m}$ (Scheme 4).

Blackmond 课题组 ${ }^{[51 \sim 5 \mathrm{~m}]}$ 发现脯氨酸催化的醛的 $\alpha$ 氨化反应有着自诱导效应，将事先生成的产物分离加入 到原料的混合物中, 可以加快反应速率，此外将叔胺加 入到反应体系中产物的对映选择性会发生翻转. 


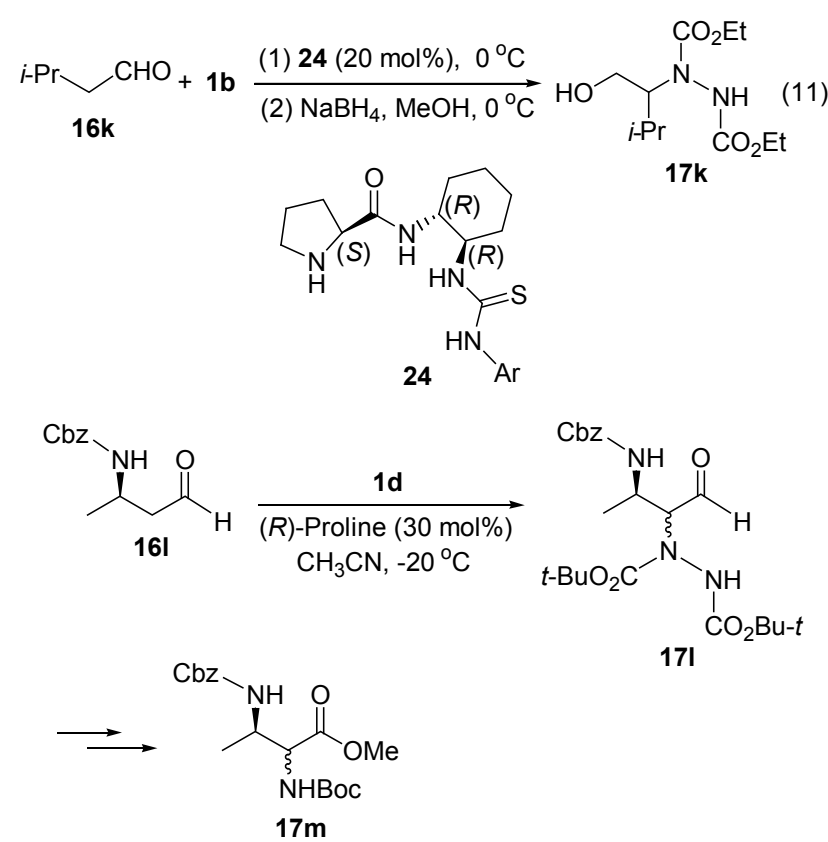

Scheme 4

有趣的是醛与偶氮二甲酸酯除发生 $\alpha$-氨化反应外, Lee 等 ${ }^{[6 a]}$ 报道了在铑催化剂下醛与偶氮二甲酸酯发生氢 酰化反应生成酰基肼 17n (Eq. 12). 该反应对多种醛(脂 肪饱和醛、不饱和醛、芳醛)都适合, 芳醛的产率相对较 低.

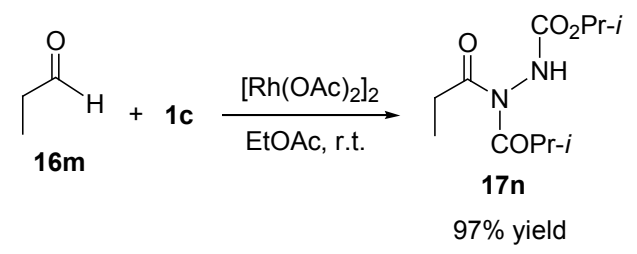

Lee 等还发现 Eq. 12 可用来合成大环化合物 ${ }^{[6 b]}$ (Scheme 5).
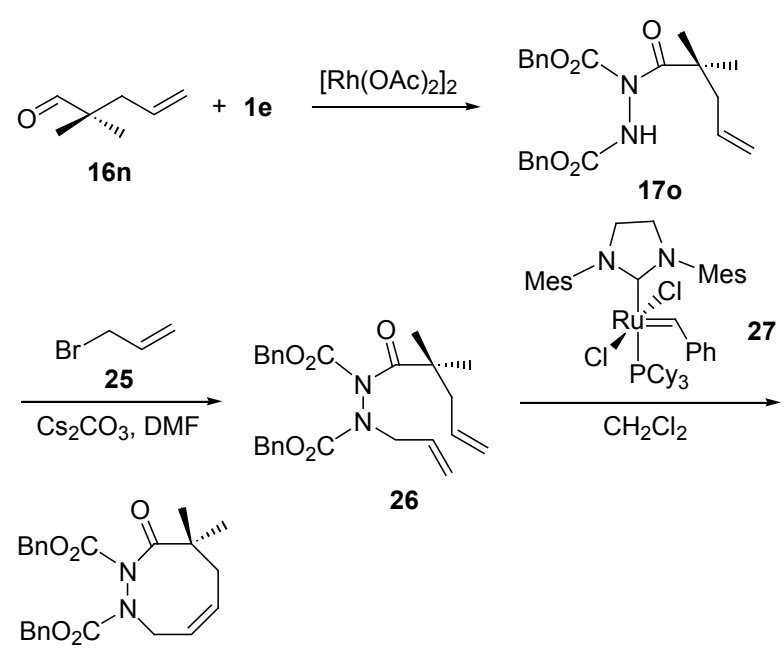

26

28

Scheme 5
2009 年 Headley 等 ${ }^{[6 c]}$ 报道了在离子液体中, 无金属 催化剂存在下醛与偶氮二甲酸酯的氢酰化反应，产率可 高达 99\%。

\section{2 酮酯氨化}

在使用脯氨酸作醛的氨化之前，双噁唑啉铜络合物 曾被用于烯醇化酮与偶氮二甲酸酯的氨化反应 ${ }^{[3 \mathrm{f}]}$. 2002 年 Jørgensen 课题组首先实现了双噁唑啉铜络合物催化 剂 $\left[(S)-\mathrm{Ph}-\mathrm{BOX}-\mathrm{Cu}(\mathrm{OTf})_{2}\right](29)$ 对 $\alpha$-酮酯 ${ }^{[7 \mathrm{a}]}$ 的催化氨化反 应(Eq. 13), 随后将此催化剂应用于 $\beta$-酮酯 ${ }^{[7 b]}$ 的氨化反 应(Eq. 14), 产物经过处理可获得 $\beta$-氨基- $\alpha$-差基酯衍生 物和 $\beta$-差基- $\alpha$-氨基酸衍生物.

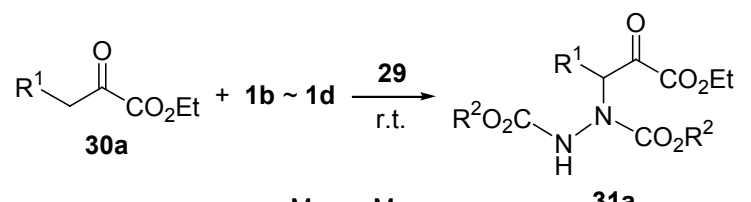

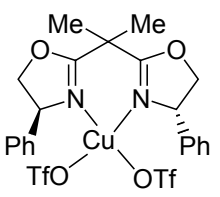

$$
\begin{aligned}
& \text { 31a } \\
& 29
\end{aligned}
$$

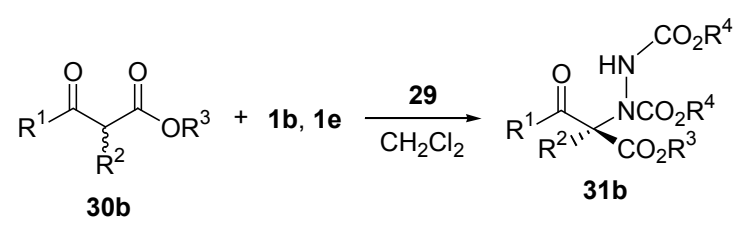

2005 年 Jørgensen 课题组 ${ }^{[7 c]}$ 又报道了使用双噁唑啉 锌络合物 32 催化剂实现 $\beta$-酮膦酸酯的氨化反应, 产物 经过相应的处理则可得到 $\beta$-羟基- $\alpha$-氨基膦酸衍生物 (Eq. 15).

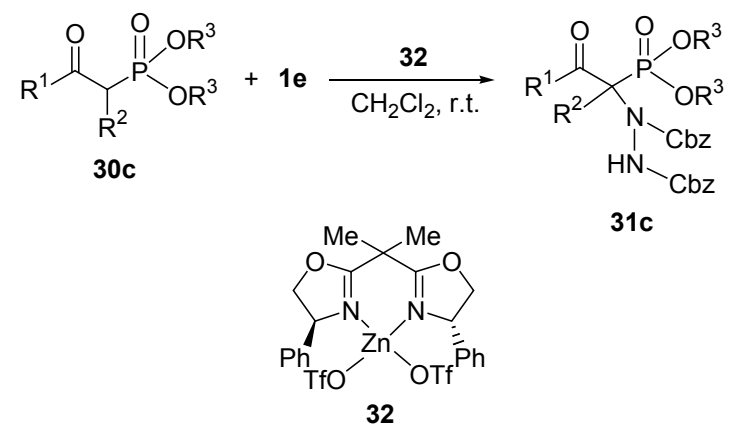

Kumagai 和 Shibasaki ${ }^{[7 \mathrm{~d} \sim 7 \mathrm{f}]}$ 报道了使用镧络合物作 催化剂催化氨化琥珀酰亚胺 30d, 该方法可用来合成一 种潜在糖醛还原酶抑制剂 AS-3201 (33). 他们首先使用 的是 $\mathrm{La}(\mathrm{OPr}-i)_{3}$ 与胺类配体形成的络合物作为催化剂 ${ }^{[7 \mathrm{~d}]}$ (Scheme 6), 但 $\mathrm{La}(\mathrm{OPr}-i)_{3}$ 在使用过程中有许多不便之 处, 随后他们便对该反应的催化剂进行重新选择并改 进, 发现 $\mathrm{La}\left(\mathrm{NO}_{3}\right)_{3} \cdot 6 \mathrm{H}_{2} \mathrm{O}$ 非常适合这个反应 ${ }^{[7 \mathrm{f}]}$. 
<smiles>CCOC1CC(=O)NC1=O</smiles>

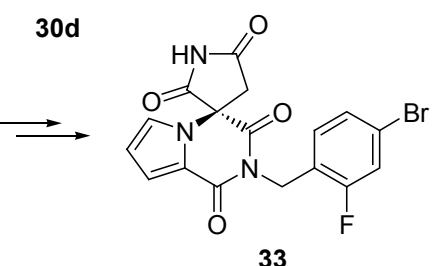

Scheme 6

2007 年 Vallribera 及其合作者 $\left.{ }^{[79]}\right]$ 报道了使用三氟甲 磺酸锞与双噁唑啉络合物 34 作催化剂催化氨化 2-氧代 环戊烷甲酸酯(Eq. 16).

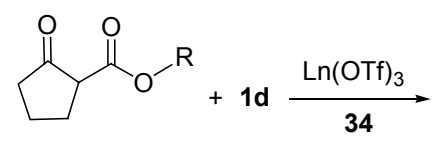

30e $\mathrm{R}=t-\mathrm{Bu}$ 30f $R=$ 1-Adamanthyl +1d $\underset{\text { Base, solvent, } 0{ }^{\circ} \mathrm{C}}{\stackrel{\text { LaPr- })_{3} \text {, ligand }}{\longrightarrow}}$

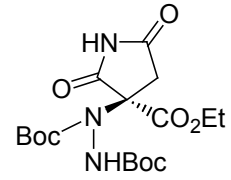

31d<smiles>CCCOC(=O)C1(N(C(=O)OCC)C(=O)OCC)CCCC1=O</smiles>

31e $\mathrm{R}=t-\mathrm{Bu}$

31f $R=$ 1-Adamanthyl<smiles>CC(C)C1COC(c2cccc(C3=N[C@@H](C(C)C)CO3)n2)=N1</smiles>

34

此外 Terada 等 ${ }^{[7 h]}$ 使用手性胍, Maruoka 等 ${ }^{[7]}$ 使用季 磷盐, Rawal 等 ${ }^{[7]}$ 使用手性方形酰胺(Squaramide)作氢键 催化剂, Kim 等 ${ }^{[7 \mathrm{k}, 71]}$ 使用手性钯, 双官能化的硫脲, Schneider 等 $[7 \mathrm{~m}]$ 使用双吡啶铜作催化剂均实现 $\beta$-酮酯的 高对映选择性氨化.

\section{$1.3 \alpha$-取代- $\alpha$-氭基羧酸酯的氨化}

在取得了对醛酮的 $\alpha$-氨化反应成功之后, Jørgensen 课题组 ${ }^{[8 a} 2004$ 年又实现了对 $\alpha$-取代- $\alpha$-氰基羧酸酯的氨 化反应(Eq. 17).

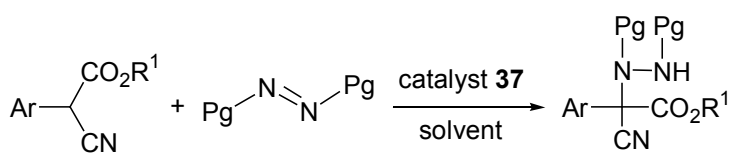

$35 a$ $\mathrm{Pg}=\mathrm{CO}_{2}$ Et. Troc, $\mathrm{Cbz}, \mathrm{Boc}$

$36 a$<smiles>CCC12CCN(CC1)[C@H](c1ccnc3ccc(O)cc13)C[C@H]2C</smiles>

37

不久 Deng 等 ${ }^{[8]}$ 使用 QD-2C/Q-2C (Scheme 7)作催

化剂高产率，高对映选择性实现了 $\alpha$-芳基- $\alpha$-氧基乙酸 乙酯与偶氮二甲酸酯的氨化反应.<smiles>C=CC1C2CCN1C(Cc1ccnc3ccc(O)cc13)C2C(=O)OC(C)(C)C</smiles>

Scheme 7

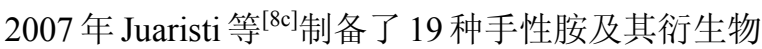
(伯胺、仲胺、叔胺)用作 $\alpha$-芳基 $\alpha$-氰基乙酸乙酯的氨化 反应，并同两种天然胺的衍生物作了对比，从而找到结 构简单的有机胺催化剂替代结构复杂的天然手性胺催 化剂.

最近 Ikariya 等 ${ }^{[8]}$ 使用双官能化的手性 $\mathrm{Ru} / \mathrm{Ir}$ 的胺络 合物作催化剂完成了 $\alpha$-芳基- $\alpha$-氧基乙酸乙酯的 $\alpha$-氨化 反应.

\section{$1.4 \alpha, \beta$-不饱和醛的氨化}

不对称催化多组份反应可以实现相对简单的过程 合成多个手性中心，同时由于减少了复杂的提纯，分离 操作更加适合环境友好性的原则. $\alpha, \beta$-不饱和醛易与亲 核试剂发生 Michael 加成反应得到手性化合物, 其所得 产物中羰基的 $\alpha$-碳可继续与亲电试剂反应. 2005 年 Jørgensen 课题组使用 $\alpha, \alpha$-二芳基甲硅烷基吡咯烷衍生 物 39 作催化剂, 不仅实现了使用有机催化剂催化硫醇 与 $\alpha, \beta$-不饱和醛的共轭加成(Eq. 18), 同时首次实现了硫 醇、 $\alpha, \beta$-不饱和醛以及偶氮二甲酸酯三组份之间的亲核 加成-亲电氨化反应 ${ }^{[9 a]}$, 反应高对映选择性( $\left.>99 \%\right)$ 得到 含有两个手性中心的氨基硫醇类衍生物(Scheme 8).

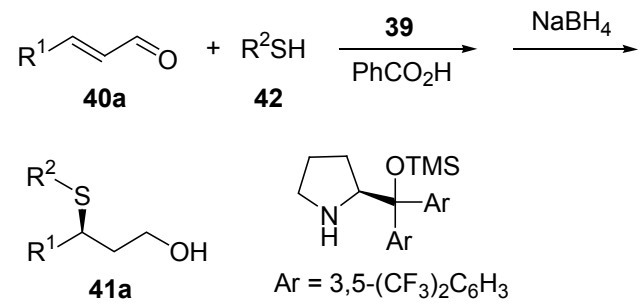

39

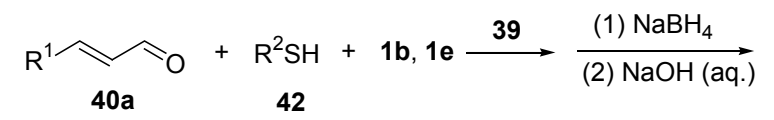<smiles>[R]OC(=O)NN1C(=O)OC[C@H]1C([R])S[R]</smiles>

Scheme 8 
2006 年 Jørgensen 课题组 ${ }^{[9 b]}$ 在原来研究的基础上使 用 $\alpha, \beta$-不饱和醛与 DEAD 进行氨化反应, 反应意外地得 到了 $\gamma$-氨化产物, 而不是预料的 $\beta$-氨化产物(Eq. 19).

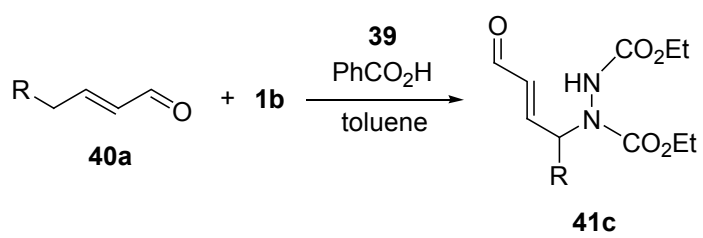

2009 年 Melchiorre 等 ${ }^{[9 c]}$ 使用手性伯胺催化剂 42, 吲 哚、硫醇作亲核试剂, 在偶氮二甲酸酯存在下与 $\alpha, \beta$-不 饱和醛完成了三组分串联反应(Eq. 20).

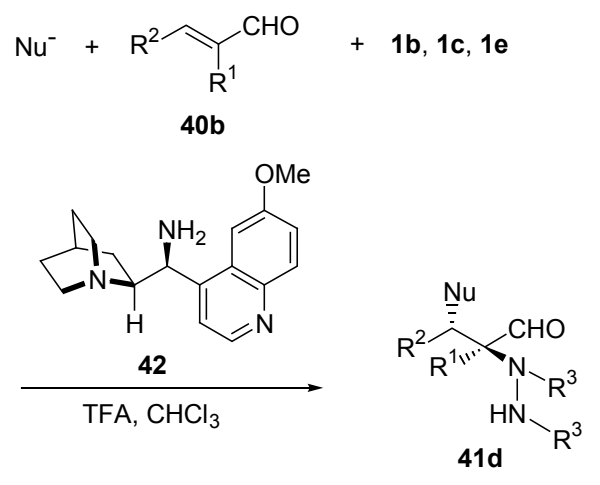

\section{5 烯丙位、碳碳双键氨化}

烯烃的氨化也是合成含氮化合物的一个重要方法. 其中有烯丙基氨化和碳碳双键的直接官能化. 1986 年 Jenner 等 ${ }^{[10 a]}$ 研究环庚三烯与双键在高压下反应时使用 环庚三烯与 DEAD 反应获得了烯丙位氨化的主要产物 (Eq. 21).

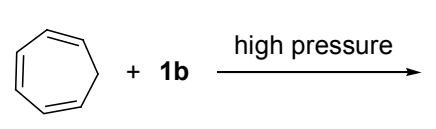

$43 a$

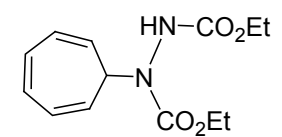

$44 a$
2005 年 Jørgensen 课题组 ${ }^{[10 b]}$ 使用 DTBAD 进行烯丙 基氨化反应的研究, 首次实现了使用金鸡纳碱类催化剂 (DHQ) $)_{2} \mathrm{PYR}$ 在无金属存在下对烯丙基位进行对映选择 催化氨化(Eq. 22).

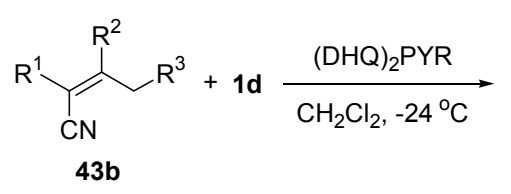<smiles>[R]C([R])=C([R])C([R])N(NC(C)(C)C)C([R])(C)C</smiles>

碳碳双键的直接氨化则吸引更多化学家的注意. 1991 年 Leblanc 等 ${ }^{[10 c]}$ 使用偶氮二甲酸二(三氯乙基)酯作 氨化试剂实现了烯烃双键碳的氨化作用, 反应产物的双
键位置会发生转移. 随后 Heathcock 及其合作者 ${ }^{[10 \mathrm{~d}]}$ 用 Lewis 酸 $\mathrm{SnCl}_{4}$ 作催化剂, DEAD 作氨化试剂实现了类似 的反应, 产物的双键位置也发生的转移, 对于不对称双 键反应更倾向于在取代基少的双键碳上发生(Eq. 23).

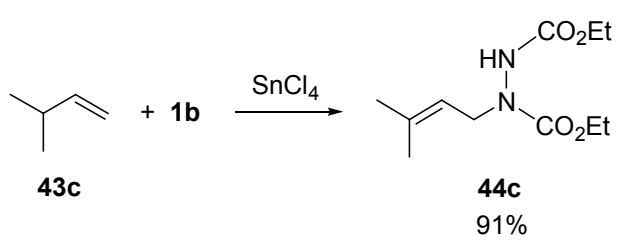

2004 年 Carreira 及合作者 ${ }^{[10 \mathrm{e}]}$ 使用金属钴催化剂 45, 在 $\mathrm{PhSiH}_{3}$ 的还原下对双键进行还原氨化反应，与 Lewis 酸作催化剂不同，反应有很高的选择性，氨化基本发生 在含取代基多的双键碳上. 有趣的是反应对羰基，羟基 和伯溴有良好的兼容性，这些基团的存在对双键碳的氨 化基本上不影响(Eq. 24).

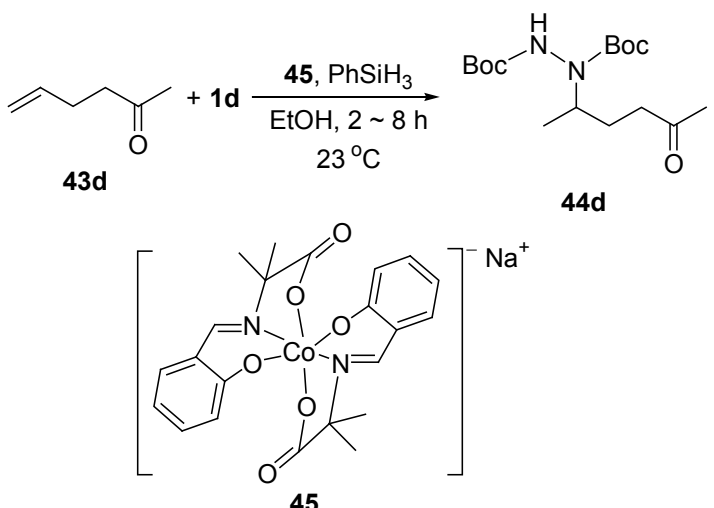

次年 Carreira 及合作者 ${ }^{[10 f]}$ 又研究了共轭双烯及烯 炔的单氨化反应. 对于共轭双烯, 产物主要是单氨化产 物(仅少量环加成产物), 得到烯丙基肼(Eq. 25); 对于烯 炔，氨化则选择性地发生在双键碳上(Eq. 26).
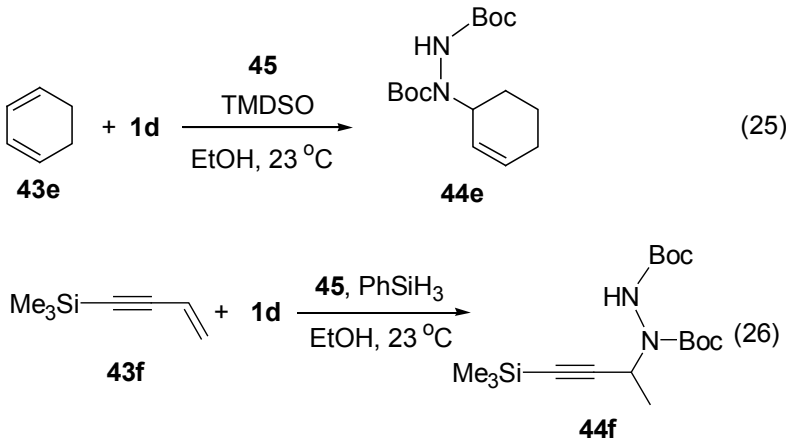

邻二胺在有机化学上也是一类很重要的化合物, 通 过烯胺双键碳的氨化有可能合成邻二胺. 2006 年 Kobayashi 及其合作者 ${ }^{[10 \mathrm{~g}]}$ 报道了烯胺甲酸酯 $43 \mathrm{~h}$ 的不对 称催化氨化反应(Eq. 27). 所得产物经水解则得到酮, 经 选择性还原则得到邻二胺. 


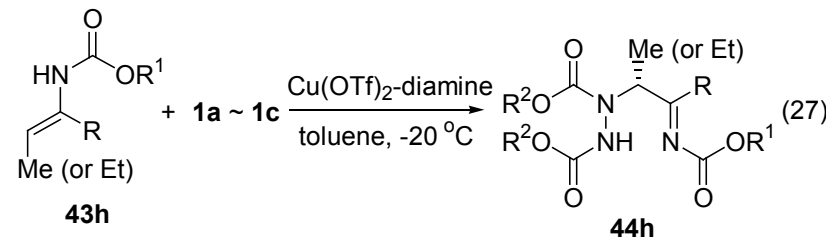

2010 年 Feng 课题组 ${ }^{[10 h]}$ 研究了 $N$-酰基烯胺的氨化 反应，相对于烯胺甲酸酯， $N$-酰基烯胺 43i 的制备更容 易. 此方法也高对映选择性地实现了 $N$-酰基烯胺的催 化氨化(Eq. 28).

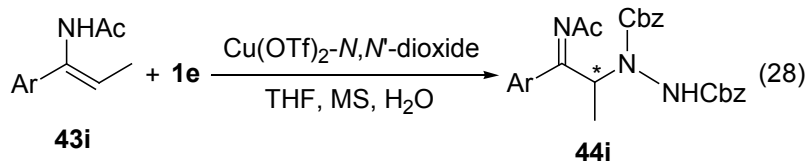

\section{6 着弪吲哚氨化}

3-氨基着弪吲哚是多种生物活性分子结构单元, 因此 合成 3-氨基羟吲哚吸引了很多化学家的兴趣. 2009 年 Chen ${ }^{[11 a]}$ 及 Zhou 等 ${ }^{[11 b]}$ 分别用(DHQD) ${ }_{2}$ PHAL 和(QD) ${ }_{2} \mathrm{P}-$ YR 作催化剂实现了 $N$-未保护的着吲哚与偶氮二甲酸二 异丙酯的氨化反应(Eq. 29).
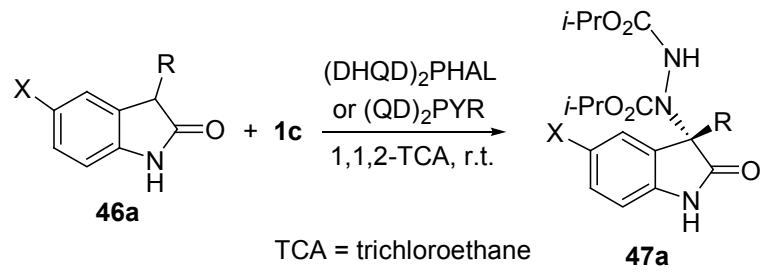

与此同时 Barbas 等 ${ }^{[11 c]}$ 实现 $N$-苠基羟吲哚与偶氮 二甲酸二乙酯的不对称氨化反应(Eq. 30).<smiles>[R]C1C(=[O+]I)N([R16]([H])([H])[H])c2ccccc21</smiles>

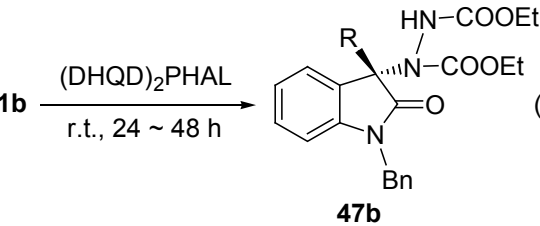

2010 年 Barbas 等 ${ }^{[11 \mathrm{~d}]}$ 对此反应进行了进一步研究, 他们设计了一类新的二聚奎尼宁类催化剂进行反应，该 催化剂对多种 3-芳基着吲哚都能适用, 包括含氟芳基羊全 吲哚.

同年 Feng ${ }^{[11 \mathrm{e}]}$, Shibasaki 等 ${ }^{[11 \mathrm{f}]}$ 分别使用钪络合物, (同种金属)双金属 Schiff 碱络合物作催化剂高产率、高 对映选择性的合成 3-氨基差弪吲哚, Shibasaki 还发现使用 单金属 Schiff碱络合物作催化剂时可以得到构型相反的 产物.

除了与羟吲哚发生氨化反应外, Shanmugam 等 ${ }^{[11 \mathrm{~g}]}$ 实现 3-取代着吲哚与偶氮二甲酸酯的环化反应合成螺
环吡唑(Eq. 31).<smiles>[R]/C(CBr)=C1/C(=O)N([R1])c2ccc([R])cc21</smiles><smiles>[R]OC(=O)N1C=C([R])C2(C(=O)N([R])c3ccc([R20])cc32)N1C([R])=O</smiles>

\section{7 芳香化合物 $\mathrm{C}$ - $\mathrm{H}$ 键的氨化、乙氧羰基化反应}

芳胺类化合物是另一类非常重要的有机化合物，芳 胺的合成也是有机合成中重要的一部分．偶氮二甲酸酯 与芳氢氨化可得到芳基肼，是合成芳胺的一条合成路 线. 1980 年 Lee 等 ${ }^{[12 a]}$ 使用吡咯与 DEAD 尝试合成 Diels-Alder 加成产物, 得到 Michael 类型双氨化产物 (Eq. 32).

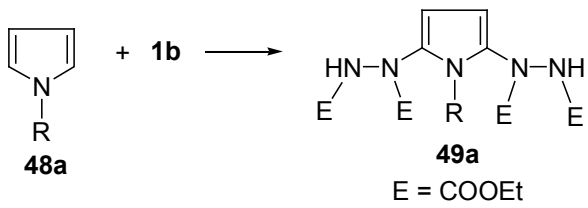

1990 年 Lee 等 ${ }^{[12 b]}$ 发现尽管上述 Michael 类型产物 是主要产物, 但改变反应条件能够得到 $[2+2+2]$ 环加 成产物，同时还能得到相当多的单氨化产物. 1993 年 Leblanc 及其合作者 ${ }^{[12 c]}$ 曾将富电子的芳环与偶氮二甲酸 二(三氯乙基)酯(1f)在高氯酸锂催化下反应得到对位氨 化产物(Eq. 33)

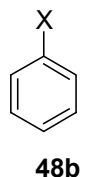

$+1 f \underset{\text { ester or acetone }}{\stackrel{3 \mathrm{~mol} / \mathrm{L} \mathrm{LiClO}_{4}}{\longrightarrow}}$

48b<smiles>[X]c1ccc(N(NC(=O)OCC(Cl)(Cl)Cl)C(=O)OCC(Cl)(Cl)Cl)cc1</smiles>

$49 b$
但此反应对一些化合物如苯甲醚，需要加热几小时 能得到比较好的结果，而二甲苯，3,5-二甲氧基苯乙酮 则不发生反应. 1994 年 Leblanc 及合作者 ${ }^{[12 \mathrm{~d}]}$ 对该方法作 了改进，他们发现 Lewis 酸 $\left(\mathrm{ZnI}_{2}\right.$ 或 $\left.\mathrm{ZnCl}_{2}\right)$ 可作为富电子 芳烃与 $1 \mathbf{f}$ 的氨化反应的活化剂, 并且发现哚也能与 1f 较好地发生氨化反应. 1995 年 Leblanc 及合作者 ${ }^{[12 \mathrm{e}]}$ 再次报道了用 $\mathrm{CF}_{3} \mathrm{SO}_{3} \mathrm{H}$ 与 $\mathrm{CF}_{3} \mathrm{COOH}$ 对该反应的的影 响, 发现它们可大幅加快氨化的反应速率.

此后 2006 年 Jørgensen 课题组 ${ }^{[12 f]}$ 也对芳烃 C-H 键 
的氨化作了研究, 他们发现萗酚 $48 \mathrm{c}$ 与偶氮二甲酸酯用 化合物 50 催化能高产率且高对映选择性地合成相应的 氨化产物(Eq. 34).

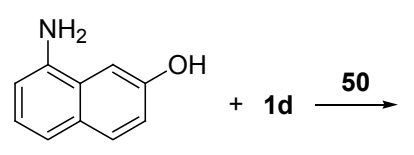

48c<smiles>CCC1CN2CCC1([C@H](O)c1ccnc3ccc(O)cc13)C[C@@H]2C</smiles>

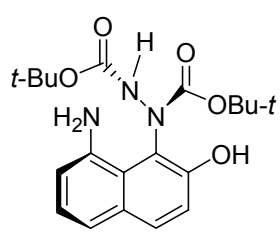

49c
Huisgen 两性离子(Huisgen zwitterion) 52a $\mathbf{5}^{[13 a]}$. Huisgen 两性离子容易与亲电试剂如异氰酸酯、异硫氰酸酯、丙 炔酸酯等发生反应生成氮杂环化合物 ${ }^{[13 a]}$ (Scheme 9).

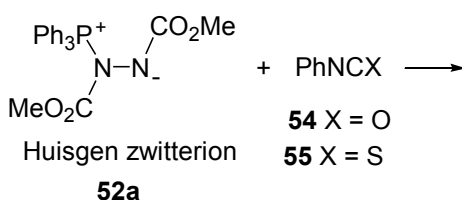

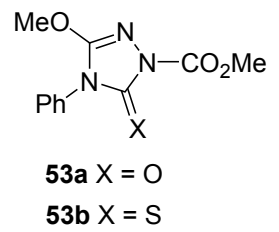<smiles>COC(=O)N([PH2+])C(=O)COC(C)=O</smiles><smiles>COC(=O)C1=CN(C(C)=O)C(C(C)=O)C1C(C)=O</smiles>

Scheme 9

他们还发现催化剂 50 可与偶氮二甲酸酯发生傅克 反应，生成的产物 49d 49e (Eq. 35)也可作为对反应 (Eq. 34)的催化剂, 且活性与原催化剂相当, 在某些反应 中催化活性甚至更高.

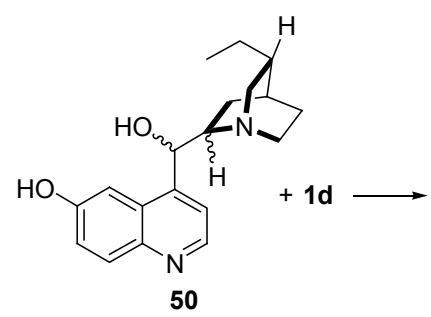

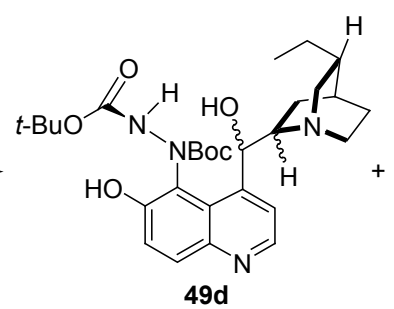<smiles>CCC1CN2CCC1[C@H]2[C@H](O)c1ccnc2ccc(O)c([N+](=O)[O-])c12</smiles>

羧酸及其衍生物是有机化学中一类广泛存在的化 合物, 从原子经济性角度来说直接将 $\mathrm{C}-\mathrm{H}$ 键官能化为 $\mathrm{C}-\mathrm{CO}_{2} \mathrm{R}$ 是非常有意义的工作. 2008 年 $\mathrm{Yu}$ 等 ${ }^{[12 \mathrm{~g}]}$ 用 $\mathrm{Pd}(\mathrm{OAc})_{2}$ 作催化剂在氧化剂及高温条件下用偶氮二甲 酸二乙酯作为 $\mathrm{C}-\mathrm{H}$ 键的氨化试剂, 结果并未得到预想 的氨化产物, 而是酯类化合物(Eq. 36).

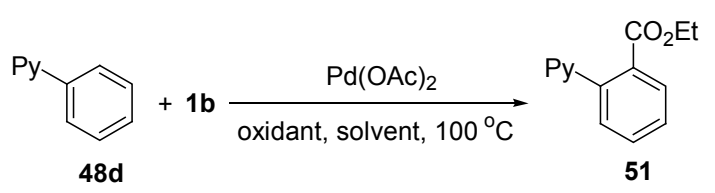

\section{Huisgen 两性离子}

三苯基膦与偶氮二甲酸酯反应得到的两性中间体， 是由 Huisgen 确定其正确结构的, 因此通常也称之为

Huisgen 两性离子在有机反应中重要的应用是 Mitsunobu 反应 ${ }^{[1]}$. Kolasa 和 Miller $^{[13 b]}$ 曾报道了 Mitsunobu 反应条件下 $\alpha$-羟基酯与 $N$-[(三氯乙氧基)羰 基]- $O$-苠基差胺的反应，反应除得到 Mitsunobu 产物外， 还得到 $\alpha$-羊基酯与 Huisgen 两性离子反应的产物 53d (Eq. 37)<smiles>COC(=O)C(O)c1ccccc1</smiles>

2003 年 Liu 等 ${ }^{[13 c]}$ 报道了 Huisgen 两性离子与含 $\alpha-\mathrm{H}$ 的酮反应，能方便得到乙烯基肼 53e (Scheme 10).
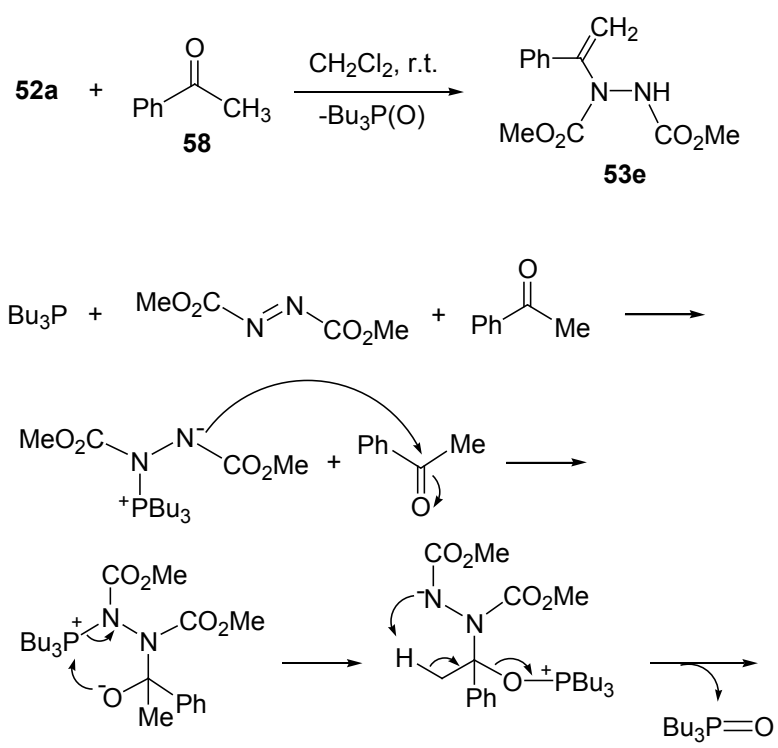<smiles>C=C(c1ccccc1)N(NC(C)=O)C(C)=O</smiles>

Scheme 10 
2005 年 Lee 等 ${ }^{[13 \mathrm{~d}]}$ 也报道了此反应，同时他们也报 道了 Huisgen 两性离子与 $\alpha$-酮酯、 $\alpha$-二酮、醛反应制备 氧杂吡唑 53f、二肼 53h 等(Eq. 38).

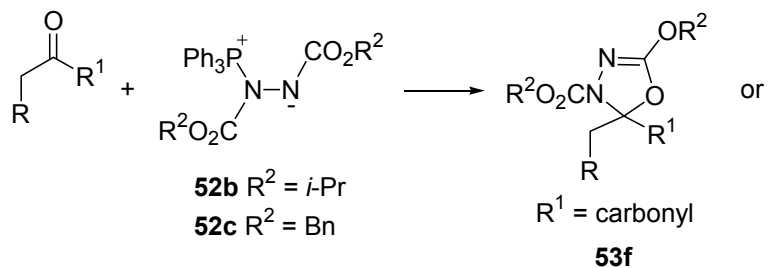

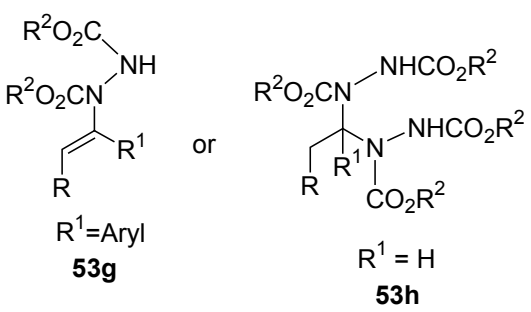

同年 Nair 课题组 ${ }^{[13 \mathrm{e}]}$ 单独研究二芳基-1,2-二酮与 Huisgen 两性离子的反应时得到与 Lee 等 ${ }^{[13 \mathrm{~d}]}$ 不相同的结 果, 反应经历了一新的重排反应得到腙 $53 \mathbf{i}$ (Eq. 39).

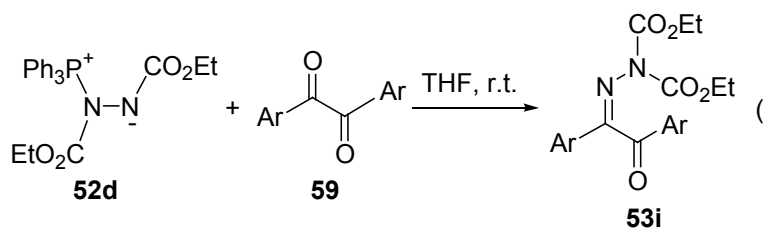

接着 Nair 课题组 ${ }^{[13 f]}$ 研究了 Huisgen 两性离子与 3甲氧基苯醌 60 、吲哚二酮的 61 反应. 反应可制得 1,2,3苯并氧杂二唑 54j 及螺环氧杂二唑 54k (Scheme 11).<smiles>CCOC(=O)N1Oc2c(C(C)(C)C)cc(C)c(OCC)c2N1C(=O)OCC</smiles>
53j

$52 d+$<smiles>CC(C)CC1C(=O)C(=O)N(Cc2ccccc2)c2ccccc21</smiles><smiles>CCOC(=O)N1N=C(OCC)OC12C(=O)N(Cc1ccccc1)c1ccccc12</smiles>

Scheme 11

Girard 等 ${ }^{[13 \mathrm{~g}]}$ 报道了由水杨醛与 Huisgen 两性离子合 成腙 531 (Eq. 40), Nair 在 2007 年则报道了由芳醛合成酰 基氨基甲酸酯 $\mathbf{5 3} \mathbf{m}^{[13 \mathrm{~h}]}$ (Eq. 41).

Huisgen 两性离子还能够与缺电子的丙二烯酯 64, 65 反应生成高度官能化的吡唑 $53 \mathrm{n}$ 与吡唑啉 530 $^{[13 i]}$ (Scheme 12).

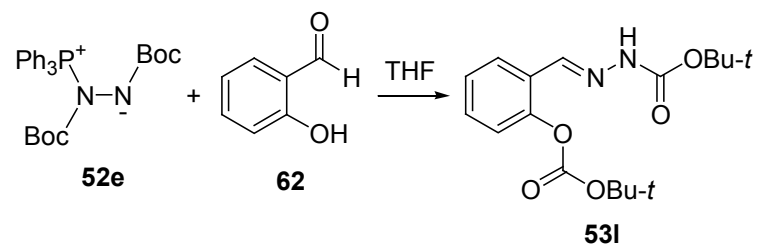

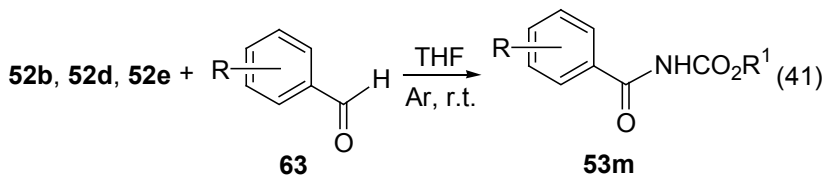

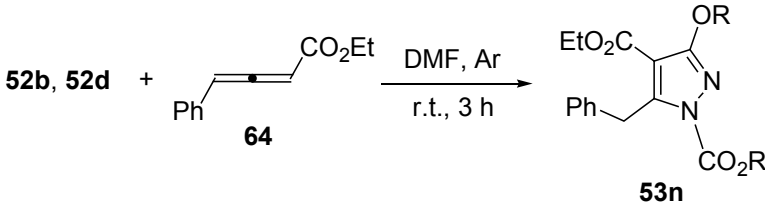

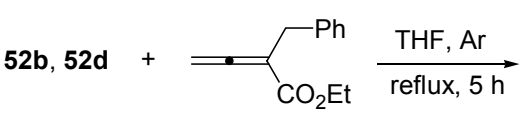

65<smiles>[R]OC1=NN(C([R])O)C(=C)C1(COc1ccco1)Cc1ccco1</smiles>

530

\section{Scheme 12}

2007 年 Nair 课题组 ${ }^{[13 j]}$ 又实现了 Huisgen 两性离子 与查尔酮的反应, 高效地合成了吡唑啉及吡唑烷衍生 物(Eq. 42).<smiles>[R]c1ccc(/C=C/C(=O)c2ccc([R])cc2)cc1</smiles>

Maron 和 Bourissou 等 ${ }^{[13 \mathrm{k}]}$ 报道了膦基硼烷 67 与 $\mathrm{DEAD}$ 反应生成 Huisgen 两性离子, 并成环得到一新的 两性离子 68 (Scheme 13).
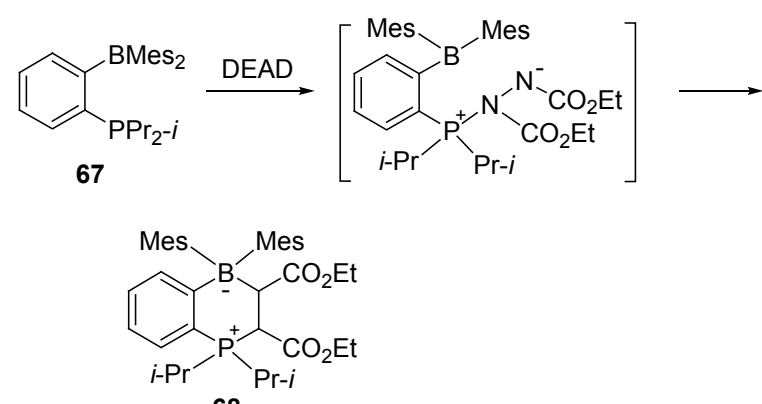

68

\section{Scheme 13}

与三苯基膦不同亚膦酸三乙基酯与 DEAD 反应则 生成膦酰肼 $\mathbf{7 0}^{[131]}$ (Eq. 43). 


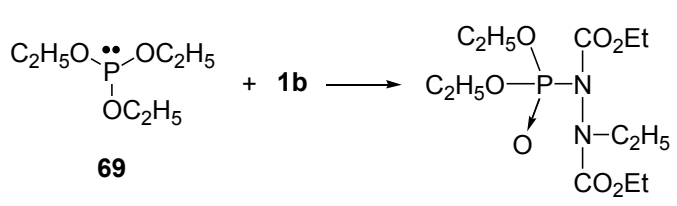

70

\section{3 环加成反应}

Diels-Alder 反应是合成环状烯的重要反应. 偶氮化 合物通常又被为二氮烯, $\mathrm{N}=\mathrm{N}$ 键又表现为与 $\mathrm{C}=\mathrm{C}$ 键相 似的性质, 偶氮二甲酸酯除了与烯烃的双键碳及烯丙基 发生氨化反应外, 也可双烯体发生 Diels-Alder 反应生成 双环偶氮化合物. 1961 年 Cohen 及合作者 ${ }^{[14 a]}$ 使用 DEAD 与环戊二烯反应合成双环偶氮化合物, 并对产物进行加 氢还原等研究(Eq. 44).

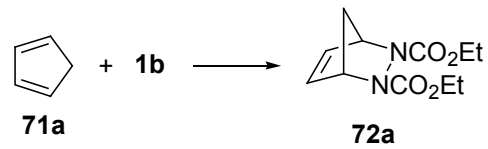

随后 Beck 及合作者 ${ }^{[14 b]}$ 报道了 2,3-二甲基-1,3-二丁 烯与 DEAD 的 Diels-Alder 环加成反应, 并研究了 2,5二甲基-2,4-己二烯与 DEAD 的氨化反应, 结果显示当双 烯的 1,4-位被高度取代时, Diels-Alder 反应在一定程度 上会被抑制.

1967 年 Anderson 及合作者 ${ }^{[14 c]}$ 使用环戊二烯缩二乙 基醚与偶氮二甲酸二甲酯反应成功地合成了 7-取代2,3-二氮双环-[2,2,1]-庚烷衍生物 73 (Scheme 14).

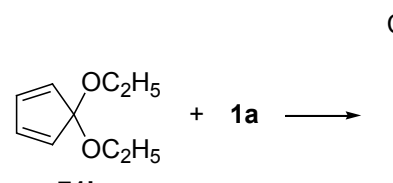

71b<smiles>COC(=O)N1C(=O)N(C(=O)OC)C2C(C(Cl)(Cl)Cl)CC1C2(Cl)Cl</smiles>

73

\section{Scheme 14}

1968 年 Alfprd 及合作者 ${ }^{[14 d]}$ 尝试用 Anderson 等 ${ }^{[14 c]}$ 的方法合成 7-取代-2,3-二氮双环-[2,2,1]-庚烷衍生物但 并未成功, 但使用亲核性更强的 1,4-酞嗪则能得到相应 的产物.

接着 Alfprd 及合作者用富烯与 DEAD 反应意外得 到了稳定的偕二醇产物 75 而不是酮(Scheme 15).

Hoornaert 等 ${ }^{[14 \mathrm{e}]}$ 和 Pindur 等 ${ }^{[14 f]}$ 分别报道了茚酤、3乙烯基吲哚与 DEAD 制得多环化合物(Eq. 45).

1999 年 Jimenez 及合作者 ${ }^{[14 \mathrm{~g}]}$ 报道了一不同于一般 Diels-Alder 反应的成环反应，他们利用 1,2-二氮-1,3-丁

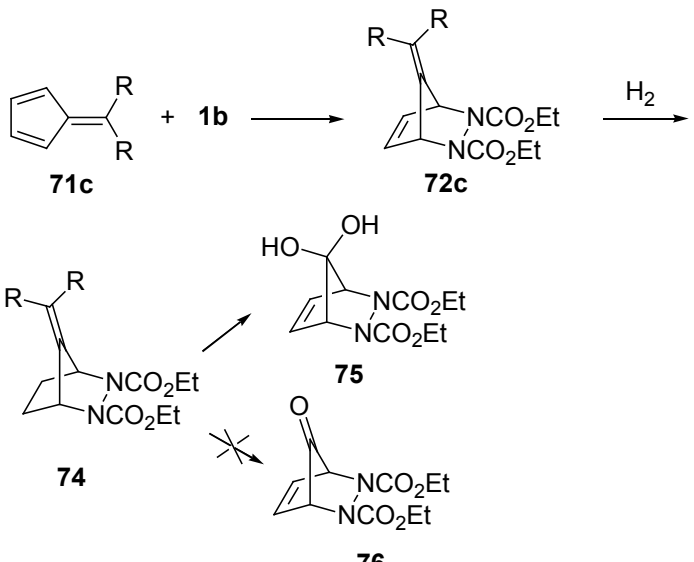

Scheme 15<smiles>CCOCN1C(OC)=CC2c3ccccc3N(C(=O)OCC)C2N1C(=O)Oc1ccccc1</smiles>

二烯长链化合物与 DEAD 在微波下实现 Hetero-DielsAlder 反应(Eq. 46).

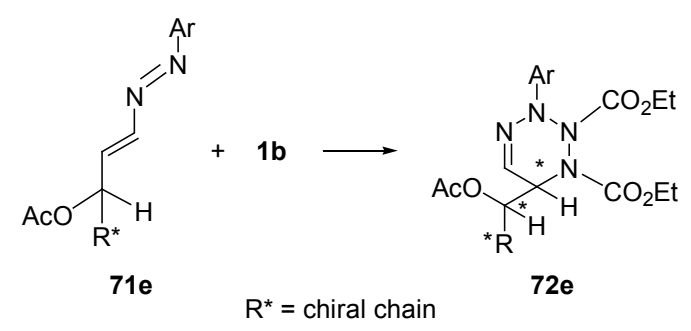

尽管 Diels-Alder 反应已经广泛用于合成环状化合 物, 但是含磷二烯作为双烯体的报道却很少. 1986 年 Minami 等 ${ }^{[14 \mathrm{~h}]}$ 报道了 1-环丁烯基二苯基氧膦作为 2-氧膦 基-1,3-丁二烯的合成子与 DEAD 合成 1,2-二乙氧基羰 基-1,2,3,6-四氢-4-二苯基氧膦哒嗪 72f (Scheme 16).

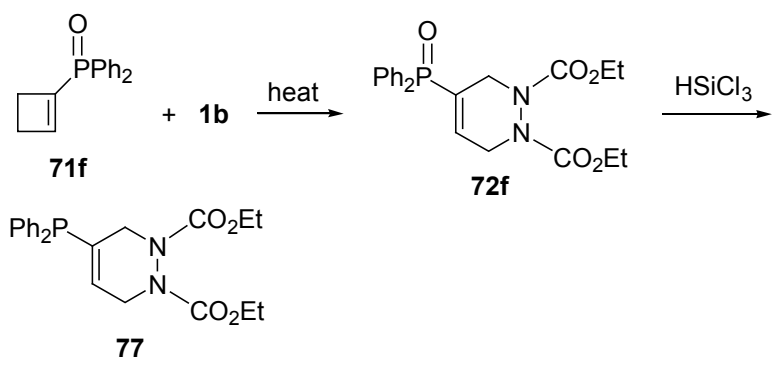

\section{Scheme 16}

2010 年 Marchand-Brynaert 等 ${ }^{[14 i]}$ 报道了 1-膦酰-1,3丁二烯 $71 \mathrm{~g}$ 与偶氮二甲酸酯反应制得了含磷化合物 $72 \mathrm{~g}$ (Eq. 47). 
<smiles>[R]OC(=O)N=[N+]=NC([R])=O</smiles>

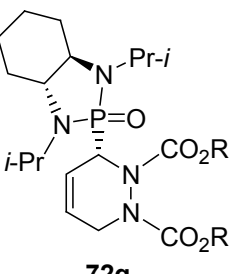

有趣的是, 偶氮二甲酸酯在环加成反应中除了能作 为亲双烯体与双烯体发生 $[4+2]$ 环加成反应外, 也能够 作为双烯体与亲双烯体发生类似的 Diels-Alder 反应. Leblanc 及合作者 ${ }^{[14 j \sim 41]}$ 报道了烯糖与偶氮二甲酸酯的 $[4+2]$ 环加成反应, 这个方法可用于制得氨基糖苷(Eq. $48)$.

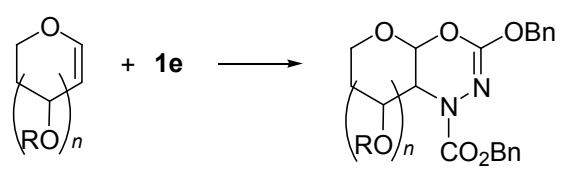

$71 \mathrm{~h}$

\section{4 合成吡唑、二氮杂环丁烷衍生物}

含氮杂环化合物是有机化学、生物有机化学中非常 重要的化合物, 许多生物活性分子中都包含有含氮杂环 结构. 吡唑、二氮杂环丁烷及其衍生物的合成一直是有 机化学家所热衰的研究, 偶氮二甲酸酯可以被用来合成 吡唑、二氮杂环丁烷及其衍生物的原料. 其中 $\mathrm{Ma}$ 等 ${ }^{[15 \sim 15 \mathrm{e}]}$ 多次发表了利用偶氮二甲酸酯、官能化的丙二 烯、卤代烷三组分反应合成吡唑、二氮杂环丁烷衍生物 的报道. 产物可随着反应底物结构的不同而有所不同.

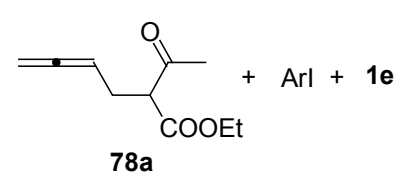

1) $\mathrm{Ph}-\mathrm{Box}, 10 \mathrm{~mol} \% \mathrm{Cu}(\mathrm{OTf})_{2}$

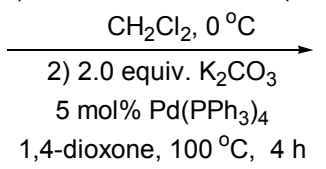<smiles>C=C(Br)C1C[C@@](COCC)(C(C)=O)N(C(=O)OCc2ccccc2)N1C(=O)OCc1ccccc1</smiles><smiles>C=C(Br)[C@@H]1C[C@@](OCC)(C(C)=O)N(C(=O)Cc2ccccc2)C1=O</smiles>

在第二步反应中通过改变钯催化剂的配体可以获 得较好的非对映选择性 ${ }^{[15 b]}$. 若反应底物是 2,3 -丙二烯 基肼 78b, 则可得到二氮杂环丁烷 $79 \mathrm{c}^{[15 \mathrm{c}]}$ (Eq. 50).

反应中若底物是 2-取代-2,3-丙二烯基肼(78c)则产 物是吡唑衍生物 $79 \mathbf{d}^{[15 \mathrm{e}]}$ (Eq. 51).

此外, 2008 年 $\mathrm{Wang}^{[15 \mathrm{f}]}$ 和 Roy 等 ${ }^{[15 \mathrm{~g}]}$ 分别利用

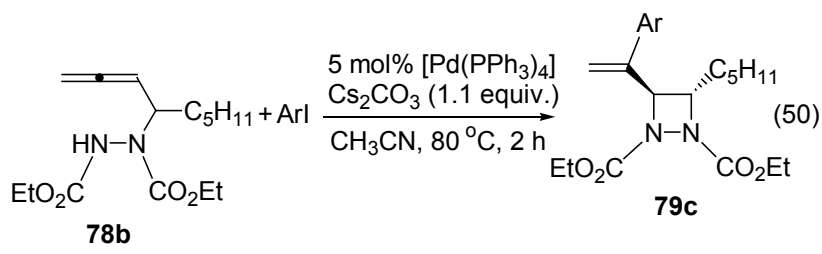

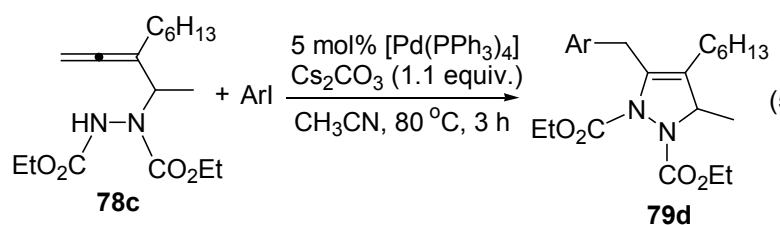

Huisgen 两性离子与氮杂环丙烷, 溴代 Baylis-Hillman 反 应产物与偶氮二甲酸酯反应制得了吡唑类衍生物 (Scheme 17).

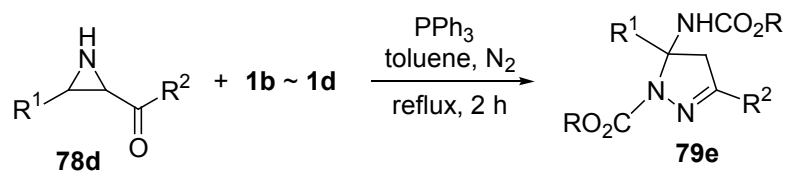

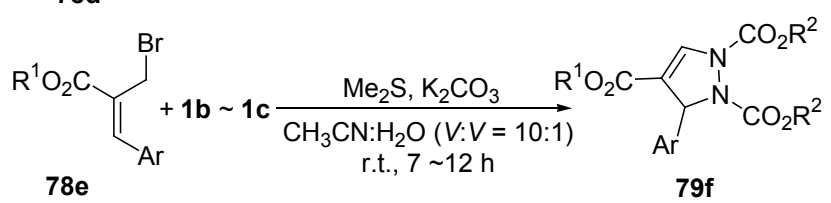

Scheme 17

\section{5 合成三唑衍生物}

1,2,4-三唑啉是一类尚未被人们完全熟知的杂环化 合物, 其生物活性很大程度上尚未被开发. 因此不少化 学家对其合成和性质产生了强烈的兴趣. 2006 年 Wang 和 $\mathrm{Xu}$ 等 ${ }^{[16 a]}$ 首次卟啉钉类催化剂 80 (Scheme 18)催化 $\alpha$ 重氮酯 81a, 亚胺 $81 \mathrm{~b}$ 及偶氮二甲酸酯三组份偶联反应 合成 1,2,4-三唑啉(Eq. 52).

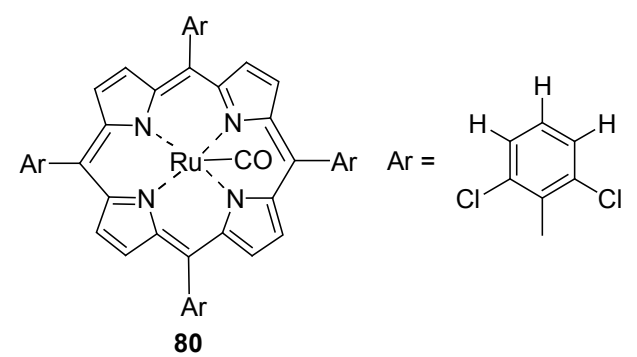

Scheme 18

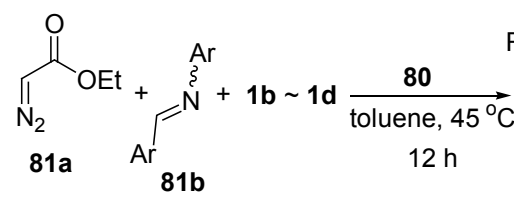

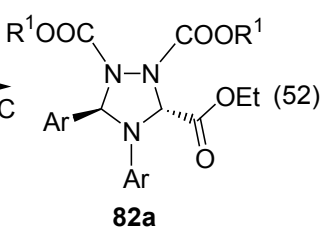


2010 年 Tepe 等 ${ }^{[16 b]}$ 使用噁唑酮与偶氮二甲酸酯在室 温下，无催化剂下成功高产率的合成了 1,2,4-三唑啉的 衍生物 $82 \mathrm{~b}$, 反应产物在碱性条件下回流即可得到三唑 82c (Scheme 19).

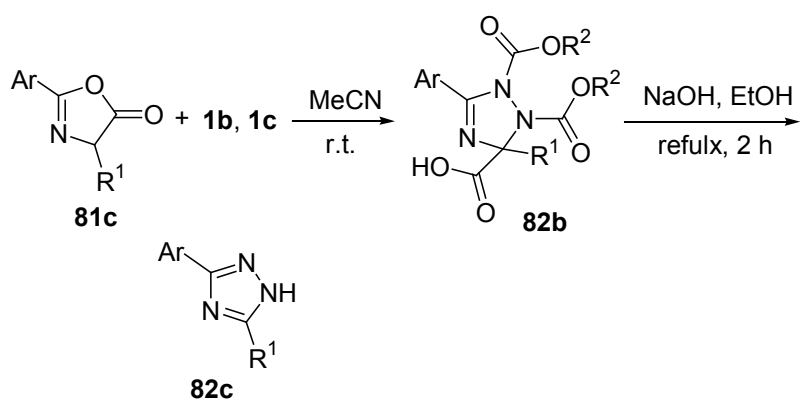

Scheme 19

同年 Wang 等 ${ }^{[16 c]}$ 用偶氮二甲酸酯与醛腙在三苯基 膦存在下反应合成 1,2,4-三唑啉 82d (Eq. 53).

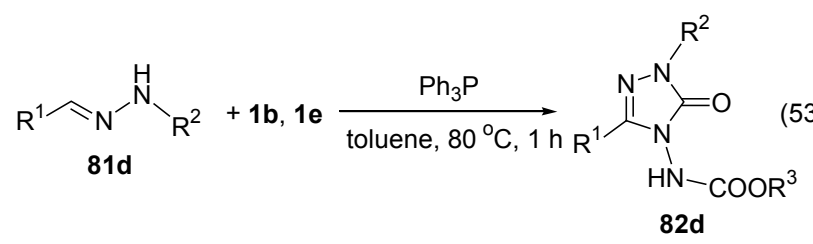

不久之后 Jørgensen 课题组 ${ }^{[16 \mathrm{~d}]}$ 也实现了用 $\alpha$-异氰基 酯与偶氮二甲酸酯生成 1,2,4-三唑啉的反应(Eq. 54).

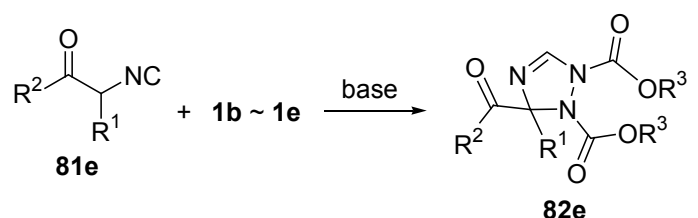

与 Tepe 等 ${ }^{[166]}$ 的方法不同此方法 C-3 位连有酯基或 是酰胺键, C-5 位则是氢原子. 2011 年 Wang 等 $^{[166]}$ 用 $\mathrm{PPh}_{3}$ 作催化剂用 2-叠氮丙烯酸酯作底物, 也同样得到了 1,2,4-三唑啉 他们发现在单独使用一种溶剂时(如甲苯, $\left.\mathrm{CH}_{3} \mathrm{CN}\right)$ 时反应并不理想, 而使用组合溶剂时 $\left[V(\mathrm{PhMe}): V\left(\mathrm{CH}_{3} \mathrm{CN}\right)=1: 1\right]$ 反应则能很好的发生(Eq. $55)$.

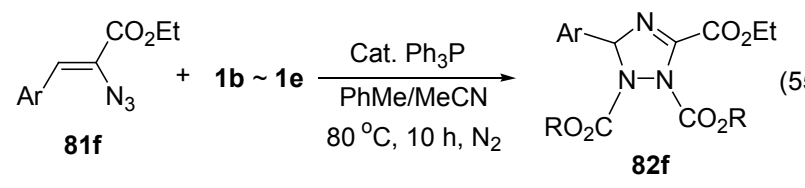

\section{6 与烯酮的反应}

尽管氮杂 $\beta$-内酰胺具有生物活性, 并在有机化学中 可作为中间体, 但只有有限的关于此类杂环化合物合成 的报道 ${ }^{[17 \mathrm{a}]}$. 其中一种合成氮杂 $\beta$-内酰胺的方法是烯䣶 与偶氮化合物的 $[2+2]$ 环加成反应.

2008 年 $\mathrm{Fu}$ 及合作者 ${ }^{[176]}$ 用他们自己设计的 4-吡咯烷 吡啶类催化剂首先实现了氮杂 $\beta$-内酰胺 84a 的不对称合 成(Eq. 56).

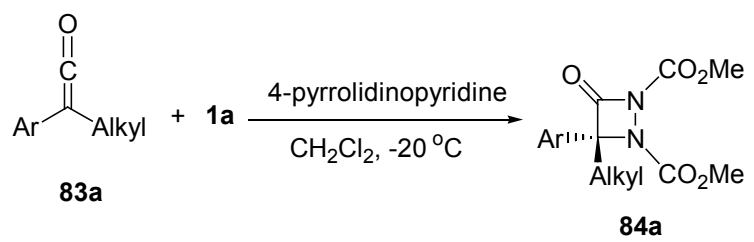

2009 年 Ye 及合作者 ${ }^{[17 c]}$ 对该反应也进行的研究, 他 们发现氮杂卡宾 $(\mathrm{NHC})$ 是一类很好的催化剂催化形成 $[2+2]$ 环加成产物, 因此将 NHC 应用此反应中, 得到令 人满意的结果.

尽管 NHC 在此反应中取得了很好的结果, 且也能 广泛用于其他反应，但对 NHC 催化反应的反应机理的 研究却很少. 2011 年 Zhu 课题组 ${ }^{[17 d]}$ 使用 NHC 作催化剂 合成氮杂 $\beta$-内酰胺并对 NHC 催化剂的机理进行了研究, 并指出对映选择性的关键步骤.

Tidwell 等 ${ }^{[17 e]}$ 报道了二(三甲基硅基)-1,2-二烯酮与 仲胺反应制得 5-氨基-3,4-二(三甲基硅基)二氢呋喃-2酮, 烯醇化后与 DEAD 反应得到开环产物(Scheme 20).<smiles>C[SiH3]C(=C=O)C(=C=O)C(C)(C)C</smiles>

$85 b$<smiles>COc1cc([GeH3])c(N(C)Cc2ccccc2)o1</smiles>

Scheme 20

\section{7 醇的氧化、硅烷化}

醇的氧化是由醇合成羰基化合物非常重要的手段, 已经发现 DEAD 有强的夺取氢原子的能力 ${ }^{[18 a]}$, 因此可 以作为醇的脱氢氧化试剂. 20 世纪 60 年代 Yoneda 及合 作者 ${ }^{[18 a, 188]}$ 就将 DEAD 用于醇的脱氢氧化反应，发现醇 被氧化成相应的羰基化合物，硫醇则被氧化成二硫化 物.

2004 年 Watanabe 课题组 ${ }^{[18 c]}$ 使用 DEAD 活化硅烷 基膦并实现了醇的硅烷化，反应选择性地发生在伯醇 上，叔醇则不发生反应(Eq. 57).

最近 Gree 等 ${ }^{[18 d]}$ 报道了 $\mathrm{DEAD}$ 与催化量的 $\mathrm{ZnBr}_{2}$ 与 


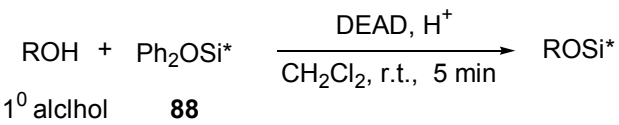

$$
\mathrm{Si}^{*}=\mathrm{SiMe}_{2} \mathrm{Bu}-t, \mathrm{SiEt}_{3}, \mathrm{Si}(\mathrm{Pr}-)_{3}
$$

醇反应将醇氧化成羰基化合物的报道，反应中 Lewis 酸 与氧原子的络合作用会促进 $\mathrm{O}-\mathrm{H}$ 键的断裂和氢的转 移, 从而加快反应(Eq. 58).

$$
\mathrm{R}_{\mathrm{R}^{2}}+\mathrm{DEAD}_{\text {Toluene, refulx }}^{\mathrm{OH}} \mathrm{R}^{1} \stackrel{\mathrm{ZnBr}}{2}_{\mathrm{R}^{2}}+2 \mathrm{H}-\mathrm{DEAD}
$$

\section{8 胺的脱氢反应}

$\mathrm{DEAD}$ 与胺反应可将与胺的氮原子相邻的 $\alpha$-氢或 $\beta$-氢脱去生成相应的亚胺或烯胺中间体. 亚胺和烯胺都 是非常重要的有机合成中间体. 2008 年我们 ${ }^{[19 a]}$ 使用叔 胺与 $\mathrm{DEAD}$ 反应生成烯胺中间体, 随后与苯磺酰叠氮反 应得到一类重要的脒化合物 91 (Eq. 59).

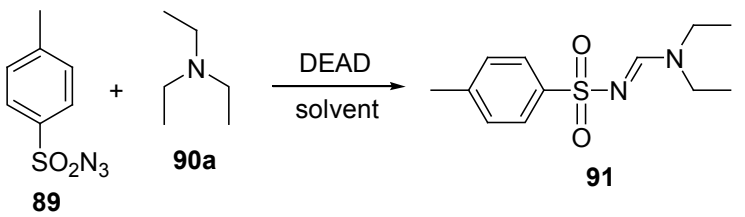

2009 年我们 ${ }^{[19 b]}$ 用 CuI 作催化剂的催化下, 用叔甲 胺与 $\mathrm{DEAD}$ 反应生成亚胺中间体, 再与端炔反应使叔胺 炔基化(Eq. 60).

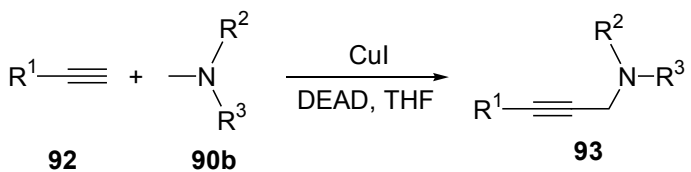

\section{9 硫氢化反应}

2010 年 Iranpoor 等 ${ }^{[20 a]}$ 发现了 DEAD 的另一个新用 途，它可用在吲哚的区域选择性硫氢化反应中(Eq. 61). 苯胺也能发生该反应，若苯胺的对位被取代反应则发生 在邻位上，但当苯胺间位被甲氧基取代时该反应不发 生.<smiles>c1ccc2[nH]ccc2c1</smiles>

$94 a$

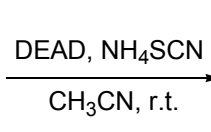

2011 年 Reddy 等 ${ }^{[20 b]}$ 用 DEAD 作促进剂实现了酮羰 基邻位碳的 $\alpha$-硫氢化反应(Eq. 62).<smiles>N#SCCC(=O)c1ccccc1</smiles>

$95 \mathrm{~b}$

\section{0 与有机锌、锆、硫、硼酸反应}

偶氮二甲酸酯中偶氮基团连有两个强吸电性基团, 因此偶氮基团通常表现为缺电子常可用作亲电试剂而 与亲核的发生氨化反应(肼化反应). 有机金属化合物, 尤其是格氏试剂一直是有机化学中常用的亲核试剂. 相 对于格氏试剂来说，有机锌卤试剂在反应过程中要相对 温和，也因此受到关注. 1998 年 Rieke 等 ${ }^{[21 a, 21 b]}$ 用有机锌 试剂与偶氮二甲酸酯发生氨化反应. 反应对酯基、氰基 有良好的兼容性(Eq. 63).

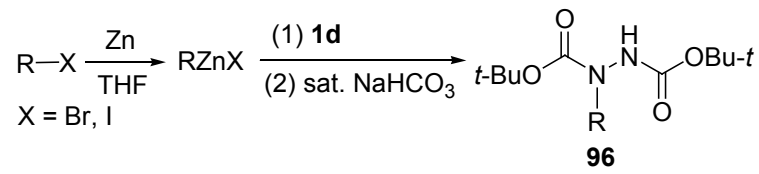

1999 年 Bertrand 等 ${ }^{[21 c]}$ 研究二乙基锌在分子间自由 基加成反应中作为链传递试剂发现将二乙基锌与 DEAD 反应可高产率地得到加成产物 97 (Eq. 64).

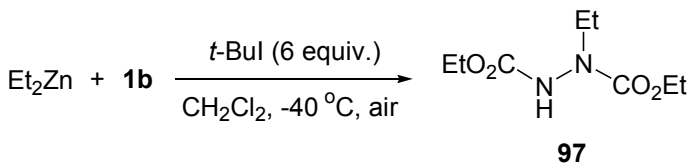

2002 年 Takahashi 等 ${ }^{[21 d]}$ 用有机锆试剂合成六员环 状化合物，成功将环戊二烯锆与 DIAD 反应制得二氮杂 苯衍生物 99 (Eq. 65).
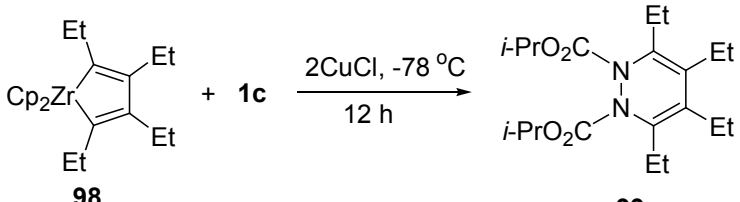

除有机锌等有机金属试剂外，一些有机非金属试剂 也能与偶氮二甲酸酯发生加成反应，硫醇、有机硼酸均 能与偶氮二甲酸酯发生反应.

1968 年 Mukaiyama 等 ${ }^{[21 e]}$ 使用硫醇与 DEAD 反应制 备二硫化物，并使 DEAD 还原成 2H-DEAD (Scheme 21).

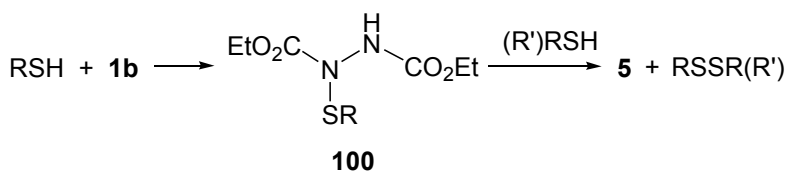

Scheme 21

1999 年 Hindsgaul 等 ${ }^{[21 f]}$ 用此法制得乙硫醇与 DEAD 的加成反应并用于与寡糖反应合成含硫寡糖.

芳基硼酸对空气、水、热不敏感，因此芳基硼酸的 应用较多。芳基硼酸与偶氮二甲酸酯反应则可以在偶氮 
基团上连接一苯基或芳基, 是合成芳基取代肼的有效且 简单的方法. 2005 年 Chatani 等 ${ }^{[21 g]}$ 使用铜盐作催化剂实 现了芳基硼酸与偶氮二甲酸酯的加成反应(Eq. 66).

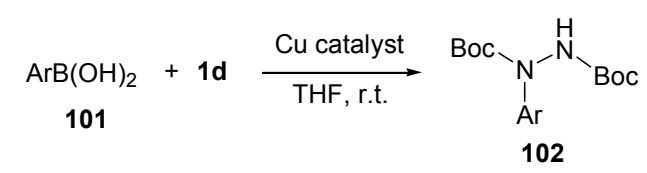

2006 年 Maeorg 等 ${ }^{[21 h]}$ 也研究此反应并将反应拓展 到非对称的偶氮二甲酸酯与芳基硼酸的反应. 同年 Muniz 等 ${ }^{[21]}$ 研究钯催化剂对偶氮双键活化, 反应中生成 钯二氮杂环甲烷, 与酸反应则能将偶氮酸酯还原成肼 (Scheme 22).<smiles></smiles>

103

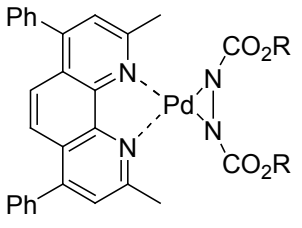

104

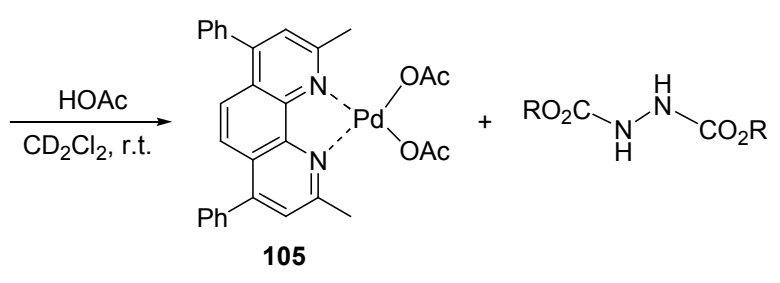

Scheme 22

随后 Muniz 等 ${ }^{[21 j]}$ 对此钯二氮杂环甲烷进行了更深 入的研究发现其可用作催化剂用于芳基嗍酸与偶氮二 甲酸酯的加成反应.

\section{1 其它反应}

DEAD 不仅可以氧化醇成醛、酮类化合物还可以选 择性氧化含硫氨基酸生成砜类化合物 $\mathbf{1 0 8}^{[22 a]}$ (Scheme 23).

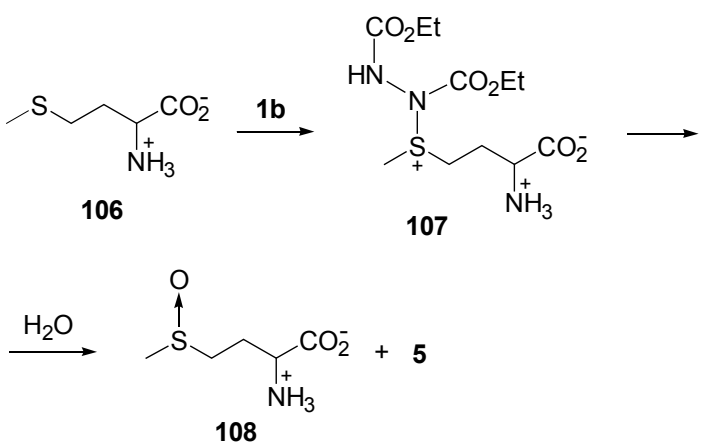

Scheme 23

2-芳基萜类化合物由于空间较大的位阻而难以发
生反应, Clarke 等 ${ }^{[22 b]}$ 报道了其与 DEAD 发生反应, 产物 经简单的水解得到多环化合物 111 (Scheme 24).

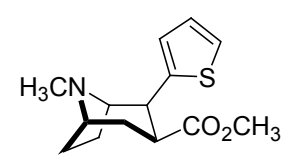

109

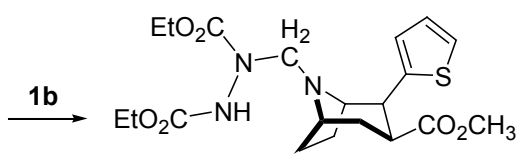

110

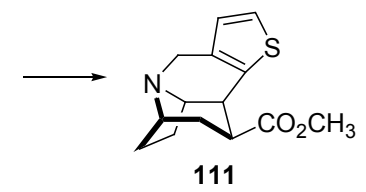

Scheme 24

Wamhof 等 ${ }^{[22 c]}$ 报道了 DEAD 与尿嘧啶类化合物反 应时经历杂环过程形成咪唑并三嗪 114 (Scheme 25).<smiles>CCOCN(C(=O)OCC)C1C(=O)N(C)C(=O)C1N(C)C(=O)N(C)CC</smiles>

112

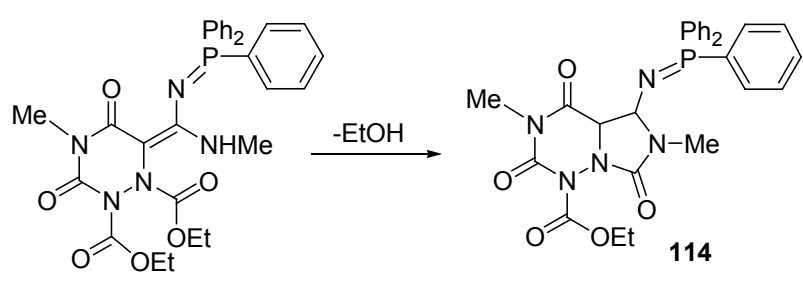
113

\section{Scheme 25}

金刚烷的衍生物可通过金刚烷桥头碳的取代反应 来获得. Eguchi 等 ${ }^{[22 \mathrm{~d}]}$ 报道了卤代金刚烷在 $\mathrm{Bu}_{3} \mathrm{SnH} /$ AIBN 存在下形成自由基, 与缺电子的烯烃如偶氮二甲 酸二乙酯、氯甲酸乙酯、异氭酸酯发生自由基加成反应, 其中仅偶氮二甲酸二乙酯与碘代金刚烷发生取代反应 生成 $\mathrm{C}-\mathrm{N}$ 键 116 (Eq. 67).

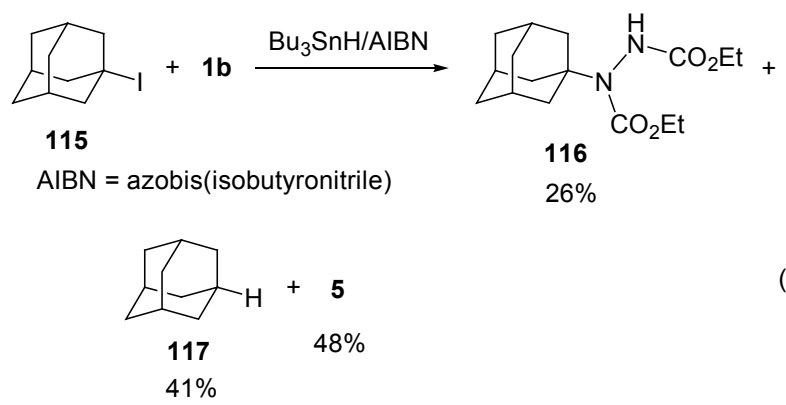

Rieber 等 ${ }^{[22 e]}$ 曾报道四环庚烷与 DEAD 或 DMAD 的 环加成反应(Eq. 68).

Sharpless 等 ${ }^{[22 f]}$ 发现与单独使用有机溶剂不同，纯 水可以加快该反应的速率甚至可以提高反应的产率, 不 


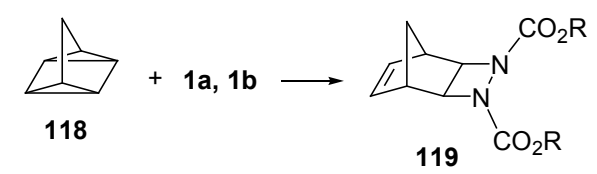

仅此反应如此，他们还发现偶氮二甲酸二(三氯乙基)酯 与烯的反应中有同样的现象存在.

最近 Zhou 等 ${ }^{[22 \mathrm{~g}}$ 报道了 $\mathrm{AgOAc}$ 催化甘氨酸 Schiff 碱与 DTBAD 反应，该反应可制得 $\alpha, \alpha$-二氨基羰基化合 物 121 (Eq. 69).

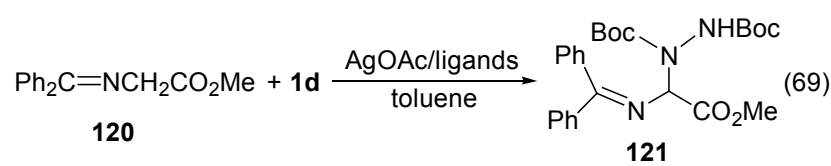

\section{2 结论与展望}

本文介绍了常用偶氮二甲酸酯的性质及其在有机 合成的常用用途，表明偶氮二甲酸酯在合成含氮化合物 特别是含氮杂环化合物等反应中具有广泛的用途. 此外 偶氮二甲酸酯与多种化合物能形成非常有用的中间体 如 Huisgen 离子, 目前虽有不少关于 Huisgen 离子的报 道 ${ }^{[13]}$, 但其性质并未被人们完全熟知, 尚存可拓展的空 间. 不仅如此, 据我们所知偶氮二甲酸酯参与的自由基 反应目前鲜有文献报道 ${ }^{[12 \mathrm{~g}, 21 \mathrm{c}, 22 \mathrm{~d}]}$. 相信偶氮二甲酸酯在 有机合成中会有越来越重要的地位.

\section{References}

[1] (a) Mitsunobu, O.; Yamada, M.; Mukaiyama, T. Bull. Chem. Soc. Jpn. 1967, 40, 935.

(b) Mitsunobu, O.; Yamada, M. Bull. Chem. Soc. Jpn. 1967, 40, 2380 .

(c) Mitsunobu, O.; Eguchi, M. Bull. Chem. Soc. Jpn. 1971, 44, 3427.

(d) Mitsunobu, O. Synthesis 1981, 1.

[2] (a) Dandapani, S.; Curran, D. P. Chem. Eur. J. 2004, 10, 3130.

(b) Dembinski, R. Eur. J. Org. Chem. 2004, 2763.

(c) But, T. Y. S.; Toy, P. H. Chem. Asian J. 2007, 2, 1340.

[3] (a) Huisgen, R.; Jakob, F. Justus Liebigs Ann. Chem. 1954, 590, 37. (b) Gennari, C.; Colombo, L.; Bertolini, G. J. Am. Chem. Soc. 1986, $108,6394$.

(c) Evans, D. A.; Britton, T. C.; Dorow, R. L.; Dellaria, J. F. J. Am. Chem. Soc. 1986, 108, 6395.

(d) Trimble, L. A.; Vederas, J. C. J. Am. Chem. Soc. 1986, 108, 6397.

(e) Evans, D. A.; Nelson, S. G. J. Am. Chem. Soc. 1997, 119, 6452. (f) Evans, D. A.; Johnson, D. S. Org. Lett. 1999, 1, 595.

(g) Yamashita, Y.; Ishitani, H.; Kobayashi, S. Can. J. Chem. 2000, 78,666 .

[4] (a) List, B. J. Am. Chem. Soc. 2002, 124, 5656.

(b) Bøgevig, A.; Juhl, K.; Kumaragurubaran, N.; Zhuang, W.; Jørgensen, K. A. Angew. Chem., Int. Ed. 2002, 41, 1790.

(c) Kumaragurubaran, N.; Juhl, K.; Zhuang, W.; Bøgevig, A.; Jørgensen, K. A. J. Am. Chem. Soc. 2002, 124, 6254.

(d) Liu, T. Y.; Cui, H. L.; Zhang, Y.; Jiang, K.; Du, W.; He, Z. Q.; Chen, Y. C. Org. Lett. 2007, 9, 3671. (e) Hayashi, Y.; Aratake, S.; Imai, Y.; Hibino, K.; Chen, Q. Y.; Yamaguchi, J.; Uchimaru, T. Chem. Asian J. 2008, 3225.

(f) Vogt, H.; Vanderheiden, S.; Brase, S. Chem. Commun. 2003, 2448.

(g) Baumann, T.; Vogt, H.; Brase, S. Eur. J. Org. Chem. 2007, 266.

(h) Baumann, T.; Bachle, M.; Hartmann, C.; Brase, S. Eur. J. Org. Chem. 2008, 2207.

(i) Hartmann, C. E.; Baumann, T.; Bachle, M.; Brase, S. Tetrahedron: Asymmetry 2010, 21, 1341.

(j) Chowdari, N. S.; Ramachary, D. B.; Barbas, C. F. Org. Lett. 2003, 5, 1685.

(k) Chowdari, N. S.; Barbas, C. F. Org. Lett. 2005, 7, 867.

(1) Suri, J. T.; Steiner, D. D.; Barbas, C. F. Org. Lett. 2005, 7, 3885.

(m) Kotrusz, P.; Alemayehu, S.; Toma, S.; Schmalz, H. G.; Adler, A. Eur. J. Org. Chem. 2005, 4904.

[5] (a) Dahlin, N.; Bøgevig, A.; Adolfsson, H. Adv. Synth. Catal. 2004, 346, 1101 .

(b) Franzen, J.; Marigo, M.; Fielenbach, D.; Wabnitz, T. C.; Kjærsgaard, A.; Jørgensen, K. A. J. Am. Chem. Soc. 2005, 127, 18296.

(c) Ding, X.; Jiang, H. L.; Zhu, C. J.; Cheng, Y. X. Tetrahedron Lett. 2010, 51, 6105.

(d) Liu, P. M.; Chang, C.; Reddy, R. J.; Ting, Y. F.; Kuan, H. H.; Chen, K. Eur. J. Org. Chem. 2010, 42.

(e) Thomassigny, C.; Prim, D.; Greck, C. Tetrahedron Lett. 2006, 47, 1117.

(f) Gosiewska, S.; Soni, R.; Clarkson, G. J.; Wills, M. Tetrahedron Lett. 2010, 51, 4214.

(g) Fu, J. Y.; Huang, Q. C.; Wang, Q. W.; Wang, L. X.; Xu, X. Y. Tetrahedron Lett. 2010, 51, 4870.

(h) Fu, J. Y.; Xu, X. Y.; Li, Y. C.; Huang, Q. C.; Wang, L. X. Org. Biomol. Chem. 2010, 8, 4524.

(i) Fu, J. Y.; Yang, Q. C.; Wang, Q. L.; Ming, J. N.; Wang, F. Y.; Xu, X. Y.; Wang, L. X. J. Org. Chem. 2011, 76, 4661.

(j) Liu, C.; Zhu, Q.; Huang, K. W.; Lu, Y. X. Org. Lett. 2011, 13, 2638.

(k) Makino, K.; Kubota, S.; Hara, S.; Sakaguchi, M.; Hamajima, A.; Hamada, Y. Tetrahedron 2009, 65, 9468.

(1) Iwamura, H.; Mathew, S. P.; Blackmond, D. G. J. Am. Chem. Soc. 2004, 126, 11770.

(m) Blackmond, D. G.; Moran, A.; Hughes, M.; Armstrong, A. $J$. Am. Chem. Soc. 2010, 132, 7598.

[6] (a) Lee, D.; Otte, R. D. J. Org. Chem. 2004, 69, 3569.

(b) Kim, Y. J.; Lee, D. Org. Lett. 2004, 6, 4351.

(c) Ni, B.; Zhang, Q. Y.; Garre, S.; Headley, A. D. Adv. Synth. Catal. 2009, 351, 875.

[7] (a) Juhl, K.; Jørgensen, K. A. J. Am. Chem. Soc. 2002, 124, 2420. (b) Marigo, M.; Juhl, K.; Jørgensen, K. A. Angew. Chem., Int. Ed. 2003, 42, 1367.

(c) Bernardi, L.; Zhuang, W.; Jørgensen, K. A. J. Am. Chem. Soc. 2005, 127, 5772.

(d) Mashiko, T.; Hara, K.; Tanaka, D.; Fujiwara, Y.; Kumagai, N.; Shibasaki, M. J. Am. Chem. Soc. 2007, 129, 11342.

(e) Mashiko, T.; Kumagai, N.; Shibasaki, M. Org. Lett. 2008, 10, 2725 .

(f) Mashiko, T.; Kumagai, N.; Shibasaki, M. J. Am. Chem. Soc. 2009, 131, 14990.

(g) Comelles, J.; Pericas, A.; Moreno-Manas, M.; Vallribera, A.; Drudis-Sole, G.; Lledos, A.; Parella, T.; Roglans, A.; GarclaGranda, S.; Roces-Fernandez, L. J. Org. Chem. 2007, 72, 2077.

(h) Terada, M.; Nakano, M.; Ube, H. J. Am. Chem. Soc. 2006, 128, 16044 . 
(i) He, R. J.; Wang, X. S.; Hashimoto, T.; Maruoka, K. Angew. Chem., Int. Ed. 2008, 47, 9466.

(j) Konishi, H.; Lam, T. Y.; Malerich, J. P.; Rawal, V. H. Org. Lett. 2010, 12, 2028.

(k) Kang, Y. K.; Kim, D. Y. Tetrahedron Lett. 2006, 47, 4565.

(1) Jung, S. H.; Kim, D. Y. Tetrahedron Lett. 2008, 49, 5527.

(m) Ghosh, S.; Nandakumar, M. V.; Krautscheid, H.; Schneider, C. Tetrahedron Lett. 2010, 51, 1860.

[8] (a) Saaby, S.; Bella, M.; Jørgensen, K. A. J. Am. Chem. Soc. 2004, 126, 8120 .

(b) Liu, X. F.; Li, H. M.; Deng, L. Org. Lett. 2005, 7, 167.

(c) Liu, Y. J.; Melgar-Fernandez, R.; Juaristi, E. J. Org. Chem. 2007, 72, 1522 .

(d) Hasegawa, Y.; Watanabe, M.; Gridnev, I. D.; Ikariya, T. J. Am. Chem. Soc. 2008, 130, 2158.

[9] (a) Marigo, M.; Schulte, T.; Franzen, J.; Jørgensen, K. A. J. Am. Chem. Soc. 2005, 127, 15710.

(b) Bertelsen, S.; Marigo, M.; Brandes, S.; Diner, P.; Jørgensen, K. A. J. Am. Chem. Soc. 2006, 128, 12973.

(c) Galzerano, P.; Pesciaioli, F.; Mazzanti, A.; Bartoli, G.; Melchiorre, P. Angew. Chem., Int. Ed. 2009, 48, 7892.

[10] (a) Jenner, G.; Papadopoulos, M. J. Org. Chem. 1986, 51, 585.

(b) Poulsen, T. B.; Alemparte, C.; Jørgensen, K. A. J. Am. Chem. Soc. 2005, 127, 11614.

(c) Leblanc, Y.; Zamboni, R.; Bernstein, M. A. J. Org. Chem. 1991, 56, 1971.

(d) Brimble, M. A.; Heathcock, C. H. J. Org. Chem. 1993, 58, 5261.

(e) Waser, J.; Carreira, E. M. J. Am. Chem. Soc. 2004, 125, 5676.

(f) Waser, J.; Gonzalez-Gomez, J. C.; Nambu, H.; Huber, P.; Carreira, E. M. Org. Lett. 2005, 7, 4249.

(g) Matsubara, R.; Kobayashi, S. Angew. Chem., Int. Ed. 2006, 45, 7933.

(h) Chang, L.; Kuang, Y. L.; Qin, B.; Zhou, X.; Liu, X. H.; Lin, L. L.; Feng, X. M. Org. Lett. 2010, 12, 2214.

[11] (a) Cheng, L.; Liu, L.; Wang, D.; Chen, Y. J. Org. Lett. 2009, 11, 3874 .

(b) Qian, Z. Q.; Zhou, F.; Du, T. P.; Wang, B. L.; Ding, M.; Zhao, X. L.; Zhou, J. Chem. Commun. 2009, 6753.

(c) Bui, T.; Borregan, M.; Barbas, C. F. J. Org. Chem. 2009, 74, 8935 .

(d) Bui, T.; Hernandez-Torres, G.; Milite, C.; Barbas, C. F. Org. Lett. 2011, 12, 5696.

(e) Yang, Z. G.; Wang, Z.; Bai, S.; Shen, K.; Chen, D. H.; Liu, X. H.; Lin, L. L.; Feng, X. M. Chem. Eur. J. 2010, 16, 6632.

(f) Mouri, S.; Chen, Z. H.; Mitsunuma, H.; Furutachi, M.; Matsunaga, S.; Shibasaki, M. J. Am. Chem. Soc. 2010, 132, 1255.

(g) Selvakumar, K.; Vaithiyanathan, V.; Shanmugam, P. Chem. Commun. 2010, 2826.

[12] (a) Lee, C. K.; Kim, S. J.; Hahn, C. S. J. Org. Chem. 1980, 45, 1692.

(b) Lee, C. K.; Ahn, Y. M. J. Org. Chem. 1990, 55, 3957.

(c) Zaltsgendler, I.; Leblanc, Y.; Bernstein, M. A. Tetrahedron Lett. 1993, 34, 2441.

(d) Mitchell, H.; Leblanc, Y. J. Org. Chem. 1994, 59, 682.

(e) Leblanc, Y.; Boudreault, N. J. Org. Chem. 1995, 60, 4268.

(f) Brandes, S.; Bella, M.; Kjærsgaard, A; Jørgensen, K. A. Angew. Chem. Int. Ed. 2006, 45, 1147.

(g) Yu, W. Y.; Sit, W. N.; Lai, K. M.; Zhou, Z. Y.; Chan, A. S. C. J. Am. Chem. Soc. 2008, 130, 3304.

[13] (a) Brunn, B. E.; Huisgen, R. Angew. Chem. Int. Ed. 1969, 8, 513. (b) Kolasa, T.; Miller, M. J. J. Org. Chem. 1987, 52, 4978. (c) Liu, Y. X.; Xu, C. F.; Liu, L. Z. Synthesis 2003, 1335.

(d) Otte, R. D.; Sakata, T.; Guzei, I. A.; Lee, D. Org. Lett. 2005, 7, 495.

(e) Nair, V.; Biju, A. T.; Abhilash, K. G.; Menon, R. S.; Suresh, E. Org. Lett. 2005, 7, 2121.

(f) Nair, V.; Biju, A. T.; Vinod, A. U.; Suresh, E. Org. Lett. 2005, 7, 5139.

(g) Girard, M.; Murphy, P.; Tsou, N. N. Tetrahedron Lett. 2005, 46, 2449.

(h) Nair, V.; Mathew, S. C.; Biju, A. T.; Suresh, E. Tetrahedron Lett. 2007, 48, 9018.

(i) Nair, V.; Biju, A. T.; Mohanan, K.; Suresh, E. Org. Lett. 2006, 8, 2213.

(j) Nair, V.; Mathew, S. C.; Biju, A. T.; Suresh, E. Angew. Chem., Int. Ed. 2007, 46, 2070.

(k) Moebs-Sanchez, S.; Bouhadir, G.; Saffon, N.; Maron, L.; Bourissou, D. Chem. Commun. 2008, 3435.

(1) Morriso, D. C. J. Org. Chem. 1958, 23, 1072.

[14] (a) Cohen, B. S. G.; Zand, R.; Steel, C. J. Am. Chem. Soc. 1961, 83, 2895.

(b) Gillis, B. T.; Beck, P. E. J. Org. Chem. 1962, 27, 1947.

(c) Allred, E. L; Anderson, C. J. Org. Chem. 1967, 32, 1874

(d) Marullo, N. P.; Alford, J. A. J. Org. Chem. 1968, 33, 2368.

(e) Lahousse, H.; Martens, H. J.; Toppet, S.; Hoornaert, G. J. J. Org.

Chem. 1982, 47, 2001.

(f) Pindur, U.; Kim, M. H.; Rogge, M.; Massa, W.; Molinier, M. J. Org. Chem. 1992, 57, 910.

(g) Avalos, M.; Babiano, R.; Cintas, P.; Clemente, F. R.; Jimenez, J. L.; Palacios, J. C.; Sanchez, J. B. J. Org. Chem. 1999, 64, 6297.

(h) Minami, T.; Chikugo, T.; Hanamoto, T. J. Org. Chem. 1986, 51, 2210 .

(i) Monbaliu, J. C.; Tinant, B.; Peeters, D.; Marchand-Brynaert, J. Tetrahedron Lett. 2010, 51, 1052.

(j) Fitzsimmons, B. J.; Leblanc, Y.; Rokach, J. J. Am. Chem. Soc. 1987, 109, 285.

(k) Fitzsimmons, B. J.; Leblanc, Y.; Chan, N.; Rokach, J. J. Am. Chem. Soc. 1988, 110, 5229.

(1) Leblanc, Y.; Fitzsimmons, B. J.; Springer, J. P.; Rokach, J. J. Am. Chem. Soc. 1989, 111, 2995.

[15] (a) Ma, S. M.; Jiao. N.; Zheng, Z. L.; Ma, Z. C.; Lu, Z.; Ye, L. W.; Deng, Y. Q.; Chen, G. F. Org. Lett. 2004, 6, 2193.

(b) Yang, Q.; Jiang, X. F.; Ma, S. M. Chem. Eur. J. 2007, 13, 9310.

(c) Cheng, X.; Ma, S. M. Angew. Chem. Int. Ed. 2008, 47, 4581.

(d) Shu, W.; Yang, Q.; Jia, G. C.; Ma, S. M. Tetrahedron Lett. 2008, $64,11159$.

(e) Cheng, X.; Ma, S. M. Chem. Commun. 2009, 4263.

(f) Cui, S. L.; Wang, J.; Wang, Y. G. Org. Lett. 2008, 10, 13.

(g) Basavaiah, D.; Roy, S. Org. Lett. 2008, 10, 1819.

[16] (a) Wang, M. Z.; Xu, H. W.; Liu, Y. G.; Wong, M. K.; Che, C. M. Adv. Synth. Catal. 2006, 348, 2391.

(b) Saleem, R. S. Z.; Tepe, J. J. J. Org. Chem. 2010, 75, 4330.

(c) Su, Y. H.; Jiang, Z.; Hong, D.; Lu, P.; Wang, Y. G.; Lin, X. F. Tetrahedron Lett. 2010, 66, 2427.

(d) Monge, D.; Jensen, K. L.; Marin, I.; Jørgensen, K. A. Org. Lett. 2011, 13, 328 .

(e) Hong, D.; Zhu, Y. X.; Lin, X. F.; Wang, Y. G. Tetrahedron 2011, 67,650 .

[17] (a) Achiwa, K.; Yamada, S. Tetrahedron Lett. 1974, 15, 1799.

(b) Berlin, J. M.; Fu, G. C. Angew. Chem., Int. Ed. 2008, 47, 7048.

(c) Huang, X. L.; Chen, X. Y.; Ye, S. J. Org. Chem. 2009, 74, 7585.

(d) Wei, D. H.; Zhu, Y. Y.; Zhang, C.; Sun, D. Z.; Zhang, W. J.; Tang, M. S. J. Mol. Catal. A: Chem. 2011, 334, 108. 
(e) Allen, A. D.; Huang, W. W.; Moore, P. A.; Far, A. R.; Tidwell, T. T. J. Org. Chem. 2000, 65, 5676.

[18] (a) Yoneda, F.; Suzuki, K.; Nitta, Y. J. Am. Chem. Soc. 1966, 88, 2328.

(b) Yoneda, F.; Suzuki, K.; Nitta, Y. J. Org. Chem. 1967, 32, 727.

(c) Hayashi, M.; Matsuura, Y.; Watanabe, Y. Tetrahedron Lett. 2004, 45, 1409.

(d) Cao, H. T.; Gree, R. Tetrahedron Lett. 2009, 50, 1493.

[19] (a) Xu, X. L.; Li, X. N.; Ma, L.; Ye, N.; Weng, B. J. J. Am. Chem. Soc. 2008, 130, 14048.

(b) Xu, X. L.; Li, X. N. Org. Lett. 2009, 11, 1027.

[20] (a) Iranpoor, N.; Firouzabadi, H.; Khalili, D.; Shahin, R. Tetrahedron Lett. 2010, 51, 3508.

(b) Reddy, B. V. S.; Reddy, S. M. S.; Madan, C. Tetrahedron Lett. 2011, 52, 1432.

[21] (a) Guijarro, A.; Rieke, R. D. Angew. Chem., Int. Ed. 1998, 37, 1679.

(b) Velarde-Ortiz, R.; Guijarro, A.; Rieke, R. D. Tetrahedron Lett. 1998, 39, 9157.

(c) Bertrand, M. P.; Feray, L.; Nouguier, R.; Perfetti, P. J. Org. Chem. 1999, 64, 9189.

(d) Takahashi, T.; Li, Y. Z.; Ito, T.; Xu, F.; Nakajima, K.; Liu, Y. H.
J. Am. Chem. Soc. 2002, 124, 1144.

(e) Mukaiyama, T.; Takahashi, K. Tetrahedron Lett. 1968, 9, 5907.

(f) Hummel, G.; Hindsgaul, O. Angew. Chem., Int. Ed. 1999, 38, 1782.

(g) Uemura, T.; Chatani, N. J. Org. Chem. 2005, 70, 8631.

(h) Kisseljova, K.; Tsubrik, O.; Sillard, R.; Maeorg, S.; Maeorg, U. Org. Lett. 2006, 8, 43.

(i) Muniz, K.; Nieger, M. Angew. Chem. Int. Ed. 2006, 45, 2305.

(j) Muniz, K.; Iglesias, A. Angew. Chem. Int. Ed. 2007, 46, 6350.

[22] (a) Axen, R.; Chaykovsky, M.; Witkop, B. J. Org. Chem. 1967, 32, 4117.

(b) Clarke, R. L.; Gambino, A. J.; Heckeler, M. L. J. Org. Chem. 1978, 43, 4589.

(c) Wamhoff, H.; Schupp, W. J. Org. Chem. 1986, 51, 2787.

(d) Ohno, M.; Ishizaki, K.; Eguchi, S. J. Org. Chem. 1988, 53, 1285 .

(e) Rieber, N.; Alberts, J.; Lipsky, J. A.; Lemal, D. M. J. Am. Chem. Soc. 1969, 91, 5668.

(f) Narayan, S.; Muldoon, J.; Finn, M. G.; Fokin, V. V.; Kolb, H. C.; Sharpless, K. B. Angew. Chem. Int. Ed. 2005, 44, 3275.

(g) Chen, Q. A.; Zeng, W.; Zhou, Y. G. Tetrahedron Lett. 2009, 50, 6866.

(Li, L.; Fan, Y. ) 